\title{
Cosmological singularities, entanglement and quantum extremal surfaces
}

\author{
A. Manu, K. Narayan and Partha Paul \\ Chennai Mathematical Institute, \\ SIPCOT IT Park, Siruseri 603103, India \\ E-mail: manu.akavoor@gmail.com, narayan@cmi.ac.in, \\ pl.partha13@gmail.com
}

ABSTRACT: We study aspects of entanglement and extremal surfaces in various families of spacetimes exhibiting cosmological, Big-Crunch, singularities, in particular isotropic AdS Kasner. The classical extremal surface dips into the bulk radial and time directions. Explicitly analysing the extremization equations in the semiclassical region far from the singularity, we find the surface bends in the direction away from the singularity. In the 2dim cosmologies obtained by dimensional reduction of these and other singularities, we have studied quantum extremal surfaces by extremizing the generalized entropy. The resulting extremization shows the quantum extremal surfaces to always be driven to the semiclassical region far from the singularity. We give some comments and speculations on our analysis.

KEYworDS: 2D Gravity, AdS-CFT Correspondence

ArXiv EPrint: 2012.07351 


\section{Contents}

$\begin{array}{llr}1 & \text { Introduction } & 1\end{array}$

2 Cosmological singularities $\quad 2$

3 Classical extremal surfaces $\quad 5$

4 Quantum extremal surfaces $\quad 12$

$\begin{array}{lll}4.1 & \text { Some time-independent backgrounds } & 14\end{array}$

$\begin{array}{lll}4.2 & \text { 2-dim cosmologies and quantum extremal surfaces } & 15\end{array}$

$\begin{array}{ll}4.3 \text { More general 2-dim cosmologies } & 19\end{array}$

$\begin{array}{lll}5 & \text { Discussion } & 20\end{array}$

$\begin{array}{ll}\text { A Further details on } t(r) \text { for classical extremal surfaces } & 22\end{array}$

B Some details on calculating $S_{\text {bulk }} \quad 24$

\section{Introduction}

Some very exciting discoveries have been made recently on the black hole information paradox [1-3], unravelled via the study of entanglement and quantum extremal surfaces: a qualitative review is [4]. Perhaps the central point is that the generalized entropy $[5,6]$ obtained by incorporating the bulk entanglement entropy of matter to the classical area of the entangling RT/HRT surface [7]-[10] makes a qualitative difference to the location of the quantum extremal surfaces, with explicit calculation possible in effective 2-dimensional models where the bulk entanglement entropy can be studied through 2-dim CFT techniques (see also the early work [11]). A noteworthy point in these studies is that apparently no information on the singularity inside the black hole and associated stringy/quantum gravity effects is necessary: this is perhaps not surprising since the near horizon region is adequately semiclassical but is striking. The interior singularity could be regarded as a cosmological, spacelike Big-Crunch singularity. In this light, or indeed independently, it is tempting to ask if quantum extremal surfaces might be used to probe cosmological, Big-Crunch or -Bang, singularities: a priori it is not clear if this makes sense since the near singularity region is expected to be rife with severe stringy/quantum gravity effects. But what one might hope is to gain some insight into how these extremal surfaces either probe or avoid such singularities, in the process learning more about entanglement and quantum extremal surfaces in general. Some interesting recent work on quantum extremal surfaces and cosmologies appears in $[12,13]$ and also e.g. [14-17]. 
In this paper we investigate aspects of entanglement and quantum extremal surfaces in certain classes of spacetimes exhibiting cosmological singularities studied first in [18]-[20]. These are time-dependent deformations of $A d S / C F T$ [21]-[24] where the bulk develops spacelike Big-Crunch singularities when the dual field theory is deformed to be on a timedependent space alongwith a time-dependent gauge coupling. Perhaps the simplest of these is the AdS Kasner spacetime. There are no horizons in these spacetimes. In [25], certain families of 2-dim cosmologies with cosmological singularities were studied in 2-dim dilaton gravity with an extra scalar which drives the dynamics in these theories. Some of these can be thought of as the dimensional reduction of the isotropic AdS Kasner and other, more general, cosmologies with Big-Crunch singularities.

First we study aspects of classical extremal RT/HRT surfaces in the higher dimensional backgrounds: due to the time dependence the surfaces also dip in the time direction (besides the holographic radial direction). By a detailed study of the extremization equations in the reliable semiclassical regime far from the singularity, we show that the classical entangling surface has only mild time dependence and bends away from the singularity. Next we study quantum extremal surfaces in the 2-dim cosmologies mentioned above, keeping in mind the key quantitative feature that the 2-dim backgrounds here allow formulating the bulk entanglement contribution in terms of well-known 2-dim CFT techniques. Assuming that the bulk matter in the region far from the singularity is approximately in the ground state is reasonable since the time variations are small there: then the bulk entanglement can be approximated using these techniques. The time-dependence inherent in these backgrounds then leads automatically to an extrapolation to the rest of the spacetime. Extremizing the resulting generalized entropy then shows that the quantum extremal surfaces are also driven to the semiclassical region far from the singularity. We discuss various features here and implications.

In section 2, we review certain aspects of these cosmological singularities and in particular the 2-dim ones in [25]. In section 3, we discuss aspects of RT/HRT surfaces in the higher dimensional cosmologies, with primary focus on the AdS Kasner case. In section 4, we discuss quantum extremal surfaces: after various generalities, we discuss some time-independent backgrounds which provide some intuition (section 4.1), and then 2-dim cosmologies with the AdS Kasner singularity in section 4.2 and more general ones in section 4.3. We close with a Discussion in section 5, and some technical details in appendices.

\section{Cosmological singularities}

Here we review some aspects of the cosmological spacetimes discussed in [25]. The higher dimensional backgrounds were studied long back as time-dependent deformations of $A d S / C F T$ in [18]-[20] towards gaining insights via gauge/gravity duality into cosmological (Big-Bang or -Crunch) singularities: further investigations on some of these appear in e.g. [26]-[29]: some reviews of cosmological singularities in string theory appear in e.g. [30, 31]. While the bulk spacetime develops a cosmological Big-Crunch (or -Bang) singularity and breaks down, the holographic dual field theory (in the $A d S_{5}$ case), living on a space that itself crunches, is subject to a severe time-dependent gauge coupling $g_{Y M}^{2}=e^{\Psi}$ 
and may be hoped to provide insight into the dual dynamics. In this case the scalar $\Psi$ controls the gauge/string coupling. Generically it was found that the gauge theory response also ends up being singular [20]. There is a large family of such backgrounds exhibiting cosmological singularities, some of which we will review below. Various other references are listed in [25].

Some of these backgrounds have the technical feature that the spatial directions are all on the same footing: this isotropy allows studying these backgrounds from a possibly simpler perspective. In [25] a dimensional reduction on the spatial part of these backgrounds was carried out, which enables recasting these backgrounds from the point of view of 2-dim dilaton gravity with a dilaton potential and an extra scalar that drives the dynamics in a nontrivial manner. A prototypical example of this is the $A d S_{D}$ Kasner spacetime [18] and the 2-dim cosmology obtained from its reduction [25]: see (2.9). More generally the higher dimensional space and its reduction ansatz are of the form

$$
d s_{D}^{2}=g_{\mu \nu}^{(2)} d x^{\mu} d x^{\nu}+\phi^{\frac{2}{d_{i}}} d \sigma_{d_{i}}^{2} ; \quad g_{\mu \nu}=\phi^{\frac{d_{i}-1}{d_{i}}} g_{\mu \nu}^{(2)}, \quad D=d_{i}+2
$$

The Weyl transformation from $g_{\mu \nu}^{(2)}$ to the 2-dim metric $g_{\mu \nu}$ ensures that the dilaton kinetic energy vanishes and the action becomes

$$
S=\frac{1}{16 \pi G_{2}} \int d^{2} x \sqrt{-g}\left(\phi \mathcal{R}-U(\phi, \Psi)-\frac{1}{2} \phi(\partial \Psi)^{2}\right),
$$

The dilaton potential $U(\phi, \Psi)$ now possibly couples the dilaton $\phi$ to $\Psi$. Certain aspects of generic dilaton gravity theories of this kind (and these 2-dim cosmological backgrounds), dimensional reduction and holography are discussed in [32]. See e.g. [33] for early discussions of 2-dim dilaton gravity in the context of 2-dim black holes as well as [34] in the context of $A d S_{2}$ holography. We obtain

$$
\begin{aligned}
& g_{\mu \nu} \nabla^{2} \phi-\nabla_{\mu} \nabla_{\nu} \phi+\frac{g_{\mu \nu}}{2}\left(\frac{\phi}{2}(\partial \Psi)^{2}+U\right)-\frac{\phi}{2} \partial_{\mu} \Psi \partial_{\nu} \Psi=0, \\
& \mathcal{R}-\frac{\partial U}{\partial \phi}-\frac{1}{2}(\partial \Psi)^{2}=0, \quad \frac{1}{\sqrt{-g}} \partial_{\mu}\left(\sqrt{-g} \phi \partial^{\mu} \Psi\right)-\frac{\partial U}{\partial \Psi}=0,
\end{aligned}
$$

as the equations of motion. These give in conformal gauge $g_{\mu \nu}=e^{f} \eta_{\mu \nu}$ :

$$
\begin{aligned}
& (r r+t t) \\
& (r r-t t) \\
& \partial_{t} \partial_{r} \phi-\frac{1}{2} f^{\prime} \partial_{t} \phi-\frac{1}{2} \dot{f} \partial_{r} \phi+\frac{\phi}{2} \dot{\Psi} \Psi^{\prime}=0, \\
& -\partial_{t}^{2} \phi-\partial_{r}^{2} \phi+\dot{f} \partial_{t} \phi+f^{\prime} \partial_{r} \phi-\frac{\phi}{2}(\dot{\Psi})^{2}-\frac{\phi}{2}\left(\Psi^{\prime}\right)^{2}=0, \\
& -\partial_{t}^{2} \phi+\partial_{r}^{2} \phi+e^{f} U=0, \\
& \left(\ddot{f}-f^{\prime \prime}\right)-\frac{1}{2}\left(-(\dot{\Psi})^{2}+\left(\Psi^{\prime}\right)^{2}\right)-e^{f} \frac{\partial U}{\partial \phi}=0, \\
& -\partial_{t}\left(\phi \partial_{t} \Psi\right)+\partial_{r}\left(\phi \partial_{r} \Psi\right)-e^{f} \frac{\partial U}{\partial \Psi}=0 .
\end{aligned}
$$

There is nontrivial dynamics in the theory (2.2) driven by the extra scalar $\Psi$. In particular there are nontrivial cosmological singularity solutions here, which were analysed in [25]. 
The power-law scaling ansatze for the 2-dim fields and the higher dimensional spacetimes, from which these can be thought of as arising from via reduction, are

$$
\phi=t^{k} r^{m}, \quad e^{f}=t^{a} r^{b}, \quad e^{\Psi}=t^{\alpha} r^{\beta} \quad \rightarrow \quad d s_{D}^{2}=\frac{e^{f}}{\phi^{\left(d_{i}-1\right) / d_{i}}}\left(-d t^{2}+d r^{2}\right)+\phi^{2 / d_{i}} d x_{i}^{2}
$$

Note that $r=0$ is the asymptotic (holographic) boundary. In the vicinity of the BigCrunch singularity, there is rapid time variation, approaching a divergence. Thus taking the time derivative terms to be dominant (dropping all the other terms) gives the near singularity behaviour described by

$$
-\partial_{t}^{2} \phi+\dot{f} \partial_{t} \phi-\frac{\phi}{2}(\dot{\Psi})^{2} \sim 0, \quad-\partial_{t}^{2} \phi \sim 0, \quad \ddot{f}+\frac{1}{2}(\dot{\Psi})^{2} \sim 0, \quad-\partial_{t}\left(\phi \partial_{t} \Psi\right) \sim 0
$$

This appears "universal": the dilaton potential $U$ governing the asymptotic behaviour of the background has disappeared. Solving these shows a "universal" subsector

$$
\phi \sim t, \quad e^{f} \sim t^{a}, \quad e^{\Psi} \sim t^{\alpha} ; \quad a=\frac{\alpha^{2}}{2}
$$

which governs the cosmological singularity. Using (2.5), various families of nontrivial 2-dim cosmologies can be found as exact classical solutions: in the vicinity of the singularity they vindicate this universal behaviour but far from this region exhibit various kinds of asymptotic data which is encoded by the dilaton potential $U$. Some noteworthy examples are:

- Flat space: $U=0$. We obtain

$$
\phi=t, \quad d s^{2}=t^{\alpha^{2} / 2}\left(-d t^{2}+d r^{2}\right), \quad e^{\Psi}=t^{\alpha},
$$

With $t=T^{1-p_{1}}$, these can be seen to be the reduction of "mostly isotropic" Kasner singularities $d s^{2}=-d t^{2}+t^{2 p_{1}} d x_{1}^{2}+t^{2 p_{2}} \sum_{i} d x_{i}^{2}$.

- AdS Kasner spacetimes: these are of the form

$$
\begin{array}{rlrl}
U & =2 \Lambda \phi^{1 / d_{i}}, \quad \Lambda=-\frac{1}{2} d_{i}\left(d_{i}+1\right), \quad p=\frac{1}{d_{i}}, & \alpha & =\sqrt{\frac{2\left(d_{i}-1\right)}{d_{i}}}, \\
d s^{2} & =\frac{R^{2}}{r^{2}}\left(-d t^{2}+d r^{2}\right)+\frac{t^{2 p} R^{2}}{r^{2}} d x_{i}^{2}, \quad e^{\Psi}=t^{\alpha}, & d_{i} p^{2}=1-\frac{1}{2} \alpha^{2},(2.9) \\
\rightarrow \phi & =\frac{t R^{d_{i}}}{r^{d_{i}}}, \quad d s^{2}=\frac{t^{\left(d_{i}-1\right) / d_{i}} R^{d_{i}+1}}{r^{d_{i}+1}}\left(-d t^{2}+d r^{2}\right), & e^{\Psi} & =t^{\sqrt{2\left(d_{i}-1\right) / d_{i}}}
\end{array}
$$

$R$ is the AdS length scale. We are suppressing an implicit Kasner scale $t_{K}$ : e.g. $t^{2 p} \rightarrow\left(t / t_{K}\right)^{2 p}$. We will reinstate this as required. The higher dimensional spacetimes and their dual field theories were studied in [18]-[20]. 
- Hyperscaling violating cosmologies: the 2-dim and higher dimensional backgrounds are of the form (2.5) with exponents and parameters:

$$
\begin{aligned}
& U(\phi, \Psi)=2 \Lambda \phi^{\frac{1}{d_{i}}} e^{\gamma \Psi}, \quad \Lambda=-\frac{1}{2}\left(d_{i}+1-\theta\right)\left(d_{i}-\theta\right), \quad \gamma=\frac{-2 \theta}{\sqrt{2 d_{i}\left(d_{i}-\theta\right)(-\theta)}}, \\
& m=-\left(d_{i}-\theta\right), \quad b=\frac{m\left(1+d_{i}\right)}{d_{i}}, \quad \beta=-m \gamma, \\
& k=1, \quad \quad \quad a=\frac{\alpha^{2}}{2}, \quad \alpha=-\gamma \pm \sqrt{\gamma^{2}+\frac{2\left(d_{i}-1\right)}{d_{i}}} .
\end{aligned}
$$

Here $\theta<0, \gamma>0$. The higher dimensional backgrounds here can be obtained as cosmological deformations of reductions of nonconformal branes down to $D$ dimensions.

There are also still more complicated hyperscaling violating Lifshitz cosmologies (with nontrivial Lifshitz exponents $z$ as well) and their reductions down to 2-dimensions which were obtained in [25]: we will not discuss them here.

In what follows we will study these cosmological backgrounds and the 2-dim cosmologies obtained from their reduction. In certain places we will find it convenient and instructive to focus on the AdS Kasner singularities (2.9) above and the corresponding 2-dim cosmologies. In the following it will be useful to note the general cosmological solutions in the form (2.5), with the 2-dim fields on the left, and the higher dimensional spacetime on the right.

\section{Classical extremal surfaces}

We would like to study the behaviour of RT/HRT surfaces in these cosmological backgrounds. The nontrivial time-dependence here in general complicates finding closed form expressions for the extremal surfaces but nevertheless various scaling results and intuition can be obtained from this study, as well as quantitative information in the semiclassical regime far from the singularity.

In the higher dim isotropic spacetime (2.5), since all $x_{i}$ are equivalent, let us consider a strip-shaped subsystem with width $l$ along the $x \in x_{i}$ direction and wrapping all other $x_{i}$ directions. The bulk extremal surface is anchored at its boundary and dips into the bulk radial direction but also in time, essentially forced by the time-dependence of the bulk cosmology. The surface will continue to be spacelike: this is a nontrivial statement in such time-dependent backgrounds. The surface parametrisation and boundary conditions are

$$
\mathcal{S} \equiv(t(r), x(r)) ; \quad \Delta x=l ; \quad t(r) \stackrel{r \rightarrow 0}{\longrightarrow} t_{0} .
$$

For simplicity, we will consider the surface to be anchored on a $t=$ const slice on the boundary. The bulk surface can dip nontrivially in time so $t(r)$ is potentially a nontrivial function starting at $t=t_{0}$ on the boundary, dipping into the bulk till some point then turning back to the boundary again at $t_{0}$ (as we will see). More generally one could consider "tilted" subsystems (with $t_{L}(0) \neq t_{R}(0)$ ): we will not consider this. The area 
functional is (note $j$ takes $D-3=d_{i}-1$ values)

$$
\begin{aligned}
S & =\frac{1}{4 G_{d_{i}+2}} \int \prod_{x_{j} \neq x}^{j \in\left(1 \ldots d_{i}-1\right)}\left(\phi^{1 / d_{i}} d x_{j}\right) \sqrt{\frac{e^{f}}{\phi^{\left(d_{i}-1\right) / d_{i}}}\left(-d t^{2}+d r^{2}\right)+\phi^{2 / d_{i}} d x^{2}} \\
& =\frac{V_{d_{i}-1}}{4 G_{d_{i}+2}} \int d r \phi \sqrt{\frac{e^{f}}{\phi^{\left(d_{i}+1\right) / d_{i}}}\left(1-\left(\partial_{r} t\right)^{2}\right)+\left(\partial_{r} x\right)^{2}} .
\end{aligned}
$$

There is no $x$-dependence so the momentum conjugate to $\partial_{r} x$ gives a conserved quantity

$$
\frac{\phi \partial_{r} x}{\sqrt{\frac{e^{f}}{\phi^{\left(d_{i}+1\right) / d_{i}}}\left(1-\left(\partial_{r} t\right)^{2}\right)+\left(\partial_{r} x\right)^{2}}}=\text { const }=A
$$

This gives

$$
\left(\partial_{r} x\right)^{2}=A^{2} \frac{\frac{e^{f}}{\phi^{\left(d_{i}+1\right) / d_{i}}}\left(1-\left(\partial_{r} t\right)^{2}\right)}{\phi^{2}-A^{2}}, \quad S=\frac{V_{d_{i}-1}}{4 G_{d_{i}+2}} \int d r \frac{e^{f / 2} \phi^{\left(3-1 / d_{i}\right) / 2}}{\sqrt{\phi^{2}-A^{2}}} \sqrt{1-\left(\partial_{r} t\right)^{2}} .
$$

For the time variable, we will need to examine the second order equation of motion: this is difficult in general, but we will discuss this later in the semiclassical regime.

To gain some intuition for the behaviour of the extremal surface, let us recall the simple familiar subcase here, of pure AdS with no time-dependence: the minimal surface here lies on a constant time slice. Then comparing with (2.5) with all $t$-exponents vanishing, we have

$$
d s^{2}=\frac{R^{2}}{r^{2}}\left(-d t^{2}+d r^{2}+d x_{i}^{2}\right), \quad\left(\partial_{r} x\right)^{2}=A^{2} \frac{e^{f} / \phi^{\left(d_{i}+1\right) / d_{i}}}{\phi^{2}-A^{2}}, \quad S=\frac{V_{d_{i}-1}}{4 G_{d_{i}+2}} \int d r \frac{e^{f / 2} \phi^{\left(3-1 / d_{i}\right) / 2}}{\sqrt{\phi^{2}-A^{2}}} .
$$

We have $\frac{e^{f}}{\phi^{\left(d_{i}-1\right) / d_{i}}}=\phi^{2 / d_{i}}=\frac{R^{2}}{r^{2}}$ so this recovers the familiar Ryu-Takayanagi AdS expressions $\left(\partial_{r} x\right)^{2}=\frac{A^{2}}{\phi^{2}-A^{2}}=\frac{A^{2} r^{2 d_{i}}}{R^{2 d_{i}}-A^{2} r^{2 d_{i}}}$ and $S \sim \frac{V_{d_{i}-1}}{G_{d_{i}+2}} \int \frac{d r \phi^{2}}{\sqrt{\phi^{2}-A^{2}}} \sim \frac{R^{d_{i}} V_{d_{i}-1}}{G_{d_{i}+2}} \int \frac{d r / r^{d_{i}}}{\sqrt{1-A^{2} r^{2 d_{i}} / R^{2 d_{i}}}}$. This shows the turning point $r_{*}$ (the deepest location till which the surface dips into the bulk) at

$$
\left(\partial_{r} x\right)^{2} \rightarrow \infty \quad \rightarrow \quad A=\phi_{*}=\frac{R^{d_{i}}}{r_{*}^{d_{i}}} ; \quad l \sim r_{*} \sim \frac{R}{A^{1 / d_{i}}} .
$$

The last scaling relation (upto numerical factors) between the width $l$ and the parameter $A$ arises from using the above expressions in the width boundary condition in (3.1). We see that as the strip width $l$ increases, $r_{*}$ increases so the surface is dipping deeper into the bulk interior (and correspondingly $A$ decreases).

In the present cosmological background, for a strip with some fixed width, the surface begins to dip into the bulk radial direction, which stops at the turning point $r_{*}$ where $\left(\partial_{r} x\right)^{2} \rightarrow \infty$. From (3.4), we have

$$
\left.\left(\partial_{r} x\right)^{2} \rightarrow \infty \Rightarrow\left(\frac{e^{f}}{\phi^{\left(d_{i}+1\right) / d_{i}}} \frac{\left(1-\left(\partial_{r} t\right)^{2}\right)}{\phi^{2}-A^{2}}\right)\right|_{r_{*}} \rightarrow \infty
$$

In this case, $\phi, e^{f}$ also have time-dependence besides $r$-dependence. However we gain some intuition from looking at the limit of small strip subsystems in a region far from strong 


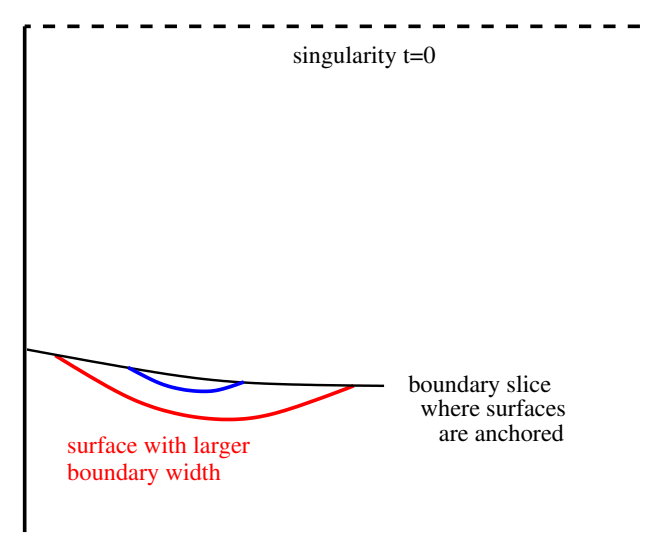

Figure 1. Cartoon of extremal surfaces in AdS Kasner, anchored on a boundary time slice (black curve). For small width (blue), the surface stays close to the boundary, while for large size (red), the surface dips deeper into the bulk. These bend away from the singularity (dotted line).

time-dependence. In this case the extremal surface can be expected to behave somewhat similar to the AdS case so the turning point will be at $\phi_{*}=A$. More pertinently $\phi$ is nonvanishing and $\partial_{r} t \ll 1$, so the only solution to the turning point equation (3.7) is $\phi_{*}=A$. As we now increase the strip subsystem size, the surface becomes "bigger" and dips further into the bulk, but continues to exist since $\phi$ is continuous. Thus this branch of extremal surfaces that is continuously connected to the AdS-like branch has turning point

$$
\left(\partial_{r} x\right)^{2} \rightarrow \infty \quad \rightarrow \quad A=\phi_{*}=\frac{t_{*}}{r_{*}^{|m|}}, \quad t_{*} \equiv t\left(r_{*}\right)
$$

We have suppressed the AdS-like lengthscale and used the scaling form $\phi=t^{k} r^{m}$ in (2.5) alongwith the universal relation $k=1$ (2.7), as well as the fact that $m=-|m|<0$ for the generic cosmological background, as noted in [25]. $m<0$ reflects the transverse area (which is the dilaton in the 2-dim description) growing towards the boundary $r \rightarrow 0$. For instance the examples (2.8), (2.9), (2.10), reviewed earlier exhibit these features explicitly.

On the face of it, the factor $\frac{e^{f}}{\phi^{\left(d_{i}+1\right) / d_{i}}}$ in (3.7) contains a factor of $t^{-\#}$ and so appears to lead to a distinct turning point localized at the singularity $t_{*}=0$ as well. However this branch of extremal surfaces appears disconnected from the branch that is continuously connected to the AdS-like branch in the region far from the singularity. If such a branch with $t_{*}=0$ exists it can only exist in the limit of infinite strip width, i.e. the IR limit, with no way to move away from $t_{*}=0$ locus (since there is no parameter like $A$ that allows deforming): this implies it is inaccessible from the classical region far from the singularity. However the region near the singularity at $t=0$ is a region where quantum gravity effects must be strong: a classical RT/HRT extremal surface localized there with no way to deform away to a well-defined classical region is unreliable. For this reason, we discard this branch of possible extremal surfaces. Finally for $\left(\partial_{r} x\right)^{2}>0$ to be well-defined, we must have $\left(\partial_{r} t\right)^{2}<1$ so $\left|\partial_{r} t\right|$ is bounded. For small strip width, the extremal surface lies on an almostconstant time slice i.e. $\left(\partial_{r} t\right)^{2} \ll 1$. These suggest that there cannot arise any divergence in (3.7) from the $\left(1-\left(\partial_{r} t\right)^{2}\right)$ term. The condition $\left(\partial_{r} t\right)^{2}<1$ is consistent with the surface 
being spacelike everywhere for our boundary conditions (3.1) (unlike e.g. [35] where the anisotropy induced by the energy flux implied that for the strip orthogonal to the flux there is a phase transition in the surfaces). Overall these arguments pin down the condition (3.8) as the relevant one for the turning point of the extremal surface (3.4) of interest.

Going with the reasonable assumption that the time direction is not doing anything singular, as described above, we will now examine the scaling of the width with $t_{*}, r_{*}, A$. It is instructive to focus on the AdS Kasner spacetime (2.9) for this purpose: (3.4) then gives

$$
\left(\partial_{r} x\right)^{2}=A^{2}\left(\frac{1}{t^{2 / d_{i}}}\right) \frac{1-\left(\partial_{r} t\right)^{2}}{\frac{t^{2}}{r^{2 d_{i}}}-A^{2}}, \quad S=\frac{V_{d_{i}-1}}{4 G_{d_{i}+2}} \int d r\left(\frac{t^{2-1 / d_{i}}}{r^{2 d_{i}}}\right) \frac{\sqrt{1-\left(\partial_{r} t\right)^{2}}}{\sqrt{\frac{t^{2}}{r^{2 d_{i}}}-A^{2}}} .
$$

These expressions can be used to explicitly see our general statements earlier. The spatial width condition (3.1) in this case gives

$$
\frac{l}{2}=\int_{0}^{r_{*}} d r\left(\partial_{r} x\right)=A \int_{0}^{r_{*}} d r \frac{e^{f / 2}}{\phi^{\left(d_{i}+1\right) / 2 d_{i}}} \sqrt{\frac{1-\left(\partial_{r} t\right)^{2}}{\phi^{2}-A^{2}}}=r_{*} \int_{0}^{1} \frac{d u}{t^{1 / d_{i}}} \frac{\sqrt{1-\left(\partial_{r} t\right)^{2}}}{\sqrt{\left(\phi / \phi_{*}\right)^{2}-1}},
$$

where $\phi=t r^{-d_{i}}$ and using (3.8). Now the integral has no nontrivial scale dependence: it has been absorbed into the $r_{*}$ factor outside. This gives the scaling, using (3.8),

$$
l \sim r_{*} ; \quad A=\frac{t_{*}}{r_{*}^{d_{i}}} \sim \frac{t_{*}}{l^{d_{i}}}
$$

This fits the expectation that as the width $l$ increases, the surface dips deeper into the bulk so the radial turning point $r_{*}$ increases. The dip in the time direction is mild at least when the surface is anchored on a time slice far from the singularity at $t=0$ : in this case the surface almost lies entirely on a constant time slice $t \sim t_{0} \gg 0$ so $t_{*} \sim t_{0}$ as well (as we discuss later). In this regime, $A \sim \frac{1}{l^{d_{i}}}$. The $u$-integral in (3.11), with each term positive, gives a positive numerical factor. The scaling $t_{*} \sim A r_{*}^{d_{i}}$ suggests that increasing $r_{*}$ implies increasing $t_{*}$. Since in this entire discussion, we restrict to one side of the singularity (the past), the range of the time variable $t$ is $t \equiv|t| \geq 0$, so increasing $t_{*}$ means bending away from the singularity at $t=0$. Similar observations were noted in [36] in a different context.

All the above arguments are reasonable as long as we are in the semiclassical regime far from the singularity: but they do not pin down $t_{*}$. More information on the time behaviour, i.e. the $t(r)$ function, is obtained by analysing the equation obtained from extremizing (3.2) with respect to the $t$-variable: this gives the $t$-equation of motion

$$
\begin{gathered}
\frac{d}{d r}\left\{\frac{\phi e^{f}\left(\partial_{r} t\right)}{\phi^{\left(d_{i}+1\right) / d_{i}} \sqrt{\frac{e^{f}}{\phi^{\left(d_{i}+1\right) / d_{i}}}\left(1-\left(\partial_{r} t\right)^{2}\right)+\left(\partial_{r} x\right)^{2}}}\right\}+\dot{\phi} \sqrt{\frac{e^{f}}{\phi^{\left(d_{i}+1\right) / d_{i}}}\left(1-\left(\partial_{r} t\right)^{2}\right)+\left(\partial_{r} x\right)^{2}} \\
+\frac{\phi\left(1-\left(\partial_{r} t\right)^{2}\right) e^{f}\left\{\dot{f}-\frac{\left(d_{i}+1\right)}{d_{i}} \frac{\dot{\phi}}{\phi}\right\}}{2 \phi^{\left(d_{i}+1\right) / d_{i}} \sqrt{\frac{e^{f}}{\phi^{\left(d_{i}+1\right) / d_{i}}}\left(1-\left(\partial_{r} t\right)^{2}\right)+\left(\partial_{r} x\right)^{2}}}=0, \quad \text { (3.12) }
\end{gathered}
$$




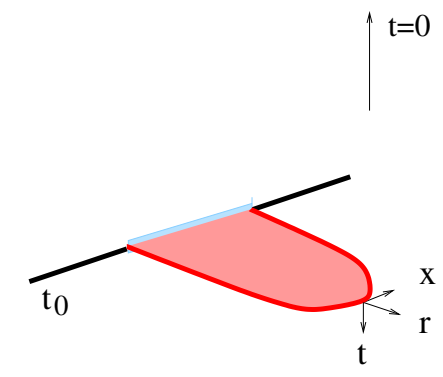

Figure 2. Cartoon of the local geometry of the extremal surface near the turning point $\left(r_{*}, t_{*}\right)$. The surface is anchored on a time slice $t_{0}$ far from the singularity at $t=0$ (not shown). It dips in the direction away from the singularity.

with $\dot{\phi}=\frac{\partial \phi}{\partial t}$ etc. Using the conserved quantity (3.4) for the $x$-variable, this simplifies. Then specialising to the AdS Kasner case, we obtain

$$
\begin{aligned}
\frac{d}{d r}\left\{\frac{\partial_{r} t}{\sqrt{1-\left(\partial_{r} t\right)^{2}}} \frac{t^{-1 / d_{i}}}{r^{d_{i}}}\right. & \left.\sqrt{t^{2}-r^{2 d_{i}} A^{2}}\right\}+\frac{t^{\left(d_{i}-1\right) / d_{i}}}{r^{d_{i}}} \sqrt{\frac{1-\left(\partial_{r} t\right)^{2}}{t^{2}-r^{2 d_{i}} A^{2}}} \\
- & \frac{1}{d_{i}} \frac{1}{t^{\left(d_{i}+1\right) / d_{i}} r^{d_{i}}} \sqrt{\left(1-\left(\partial_{r} t\right)^{2}\right)\left(t^{2}-r^{2 d_{i}} A^{2}\right)}=0 .
\end{aligned}
$$

This gives, with $t^{\prime} \equiv \partial_{r} t$,

$$
\left(1-t^{\prime 2}\right)\left(d_{i}^{2} t^{\prime}+\frac{r\left(t^{2}-A^{2} r^{2 d_{i}}\right)}{t^{3}}-\frac{d_{i} r}{t}\right)-\frac{\left(t^{2}-A^{2} r^{2 d_{i}}\right) d_{i} r t^{\prime \prime}}{t^{3}}=0 .
$$

We have suppressed the Kasner scale $t_{K}$ mentioned after (2.9): reinstating this shows that $A$ appearing above is really $A t_{K}$, so that each term above is dimensionless (we have suppressed the AdS scale $R$ : reinstating that we can rescale $t, r$ by $R$, and then the above statement on each term being dimensionless continues to hold). Now note that we have written (3.14) to emphasise that in the region far from the singularity at $t=0$, we have large $t$ so we can analyse this equation in detail. Here, as stated before, we expect that the surface will have only mild time dependence, lying almost on a constant time slice, so $\partial_{r} t \ll 1$. At the turning point, from (3.7), (3.8), we have $\phi_{*}=\frac{t_{*}}{r_{*}^{d_{i}}}=A$ and $\left(\partial_{r} x\right)^{2} \rightarrow \infty$. Then the nonzero terms in (3.14) give

$$
t_{*}^{\prime}=\frac{r_{*}}{d_{i} t_{*}}>\left.0 \quad \rightarrow \quad \frac{d r}{d x}\right|_{*}=0,\left.\quad \frac{d t}{d x}\right|_{*}=\frac{t_{*}^{\prime}}{\left(\partial_{r} x\right)_{*}}=0 .
$$

Thus the extremal surface is at a $t$-maximum at the turning point $\left(r_{*}, t_{*}\right)$. Further in its neighbourhood, we have

$$
r<r_{*}, \quad t_{*}^{\prime}>0 \quad \rightarrow \quad t(r) \sim t_{*}+t_{*}^{\prime}\left(r-r_{*}\right)<t_{*}
$$

verifying that $t_{*}$ is a local maximum (we recall that the range of $t \equiv|t|>0$ so $t$ increasing is going away from $t=0)$. This suggests the global condition $t_{*}>t_{0}$ at least for sufficiently small size (sufficiently small $r$ ), although strictly speaking that is not implied. To gain 
insight into this, we can employ perturbation theory in (3.14) to study the $t(r)$ function in the region far from the singularity. Since the time dependence there is expected to be small, we can take $t^{\prime} \ll 1$ and therefore approximate the equation (3.14) by

$$
d_{i}^{2} t^{3} t^{\prime}+r\left(t^{2}-A^{2} r^{2 d_{i}}\right)-d_{i} r t^{2}-\left(t^{2}-A^{2} r^{2 d_{i}}\right) d_{i} r t^{\prime \prime}=0,
$$

dropping $t^{\prime 2}$ in the first $\left(1-t^{\prime 2}\right)$ factor. Now a power series ansatz for $t(r)$ gives

$$
t(r)=t_{0}+\sum_{n \in \mathbb{Z}^{+}} c_{n} r^{n} \rightarrow c_{n} \sim \frac{1}{t_{0}^{\#}} .
$$

We have $t(0)=t_{0}$ as a boundary condition for anchoring the surface at the boundary. Sticking this series in (3.17) can be done numerically in Mathematica, and the $c_{n}$ can be solved for iteratively: they scale inversely with $t_{0}$. This shows for $r \leq r_{*} \lesssim t_{0}$ that $t(r)$ is indeed almost constant, with only mild variation. For example in $\operatorname{Ad} S_{5}$ Kasner $\left(d_{i}=3\right)$, we find (see appendix A for more details, specifically (A.1))

$$
t(r)=t_{0}+\frac{1}{12 t_{0}} r^{2}-\frac{1}{432 t_{0}^{3}} r^{4}+\frac{1}{7776 t_{0}^{5}} r^{6}+\left(\frac{A^{2}}{160 t_{0}^{3}}-\frac{17}{7776 \cdot 240 t_{0}^{7}}\right) r^{8}+\ldots
$$

with higher order terms further suppressed. The first few terms scale as $\frac{1}{t_{0}^{n-1}}$ so they are manifestly subleading in the regime $r_{*} \lesssim t_{0}$. The higher terms beginning with $r^{8}$ contain $A$ in their coefficients. Recall now that $A$ here is really $A t_{K}$, reinstating the Kasner scale $t_{K}$ as mentioned after (3.14). Thus $A t_{K}=t_{k} \frac{t_{*} / t_{K}}{r_{*}^{3}} \sim \frac{t_{0}}{r_{*}^{3}}$ since $t_{*} \sim t_{0}$. This means the term containing $A^{2}$ in the $r^{8}$-term is of the form $\frac{t_{*}^{2} r^{8}}{160 r_{*}^{6} t_{0}^{3}} \sim \frac{r_{*}^{2}}{160 t_{0}} \frac{r^{8}}{r_{*}^{8}} \ll t_{0}$ since $t_{*} \sim t_{0}$ and $r \leq r_{*} \lesssim$ $t_{0}$ : so this term is also suppressed compared with the leading $t_{0}$ term. Now between the two terms in the $r^{8}$ coefficient, we see that $\frac{A^{2}}{t_{0}^{3}} \sim \frac{t_{*}^{2}}{r_{*}^{6} t_{0}^{3}} \sim \frac{1}{r_{*}^{6} t_{0}}$ dominates over $\frac{1}{t_{0}^{7}}$. This is also true at higher orders where there are further terms containing $\frac{A^{2 k}}{t_{0}^{\#}}$. Analysing this further and retaining only the dominant terms in each $r^{k}$-coefficient leads to a new series (see appendix A). Evaluating this at the turning point $r_{*}$ gives (A.3): thus we manifestly see that

$$
t_{*}=t\left(r_{*}\right)>t_{0}, \quad r_{*} \lesssim t_{0},
$$

In other words, the surface bends away from the singularity at $t=0$, at least if anchored in the reliable far-region with $t_{0} \gg 0$. Using this it can be seen that $t^{\prime 2} \ll 1$ indeed so our approximation of using (3.17) instead of (3.14) is justified in this regime. Similar power series and results arise in $A d S_{4^{-}}$and $A d S_{7}$-Kasner. Thus overall, the surface function $t(r)$ starts at $t=t_{0}$ at the boundary $r=0$ and then grows, reaching a maximum value at the turning point $t=t_{*}$ bending away from the singularity. Then the surface turns around and returns to the boundary (joining the other end of the strip subsystem). This is depicted in figure 2 .

The IR limit where the strip width is large (using (3.11)) is $\frac{t_{*}}{r_{*}}=A \sim \frac{1}{d^{d_{i}}} \rightarrow 0$. In this limit, (3.17) becomes $d_{i}^{2} t^{3} t^{\prime}+r t^{2}-d_{i} r t^{2}-t^{2} d_{i} r t^{\prime \prime}=0$. Again analysing via a power series in Mathematica gives in $A d S_{5}$ Kasner $\left(d_{i}=3\right)$, we find

$$
t(r)=t_{0}+\frac{1}{12 t_{0}} r^{2}-\frac{1}{432 t_{0}^{3}} r^{4}+\frac{1}{7776 t_{0}^{5}} r^{6}-\frac{17}{1866240 t_{0}^{7}} r^{8}+\frac{247}{335923200 t_{0}^{9}} r^{10}+\ldots
$$


The series here is more delicate since the surface really has $r_{*} \rightarrow \infty$ (dipping into the bulk fully) so the entire $r$-series is important. The limit $A \rightarrow 0$ requires $A \lesssim \frac{1}{t_{0}^{2}}$ comparing with the scale $t_{0}$ : this requires $\frac{t_{0}}{r_{*}} \lesssim 1$. Thus the IR limit here is

$$
r_{*} \rightarrow \infty, \quad t_{0} \rightarrow \infty, \quad \frac{t_{0}}{r_{*}} \lesssim 1
$$

which is the reliable semiclassical regime far from the singularity. In this regime the series defining the time behaviour of the surface continues to be well-defined, albeit delicate: the surface is anchored on a slice far from $t=0$ so although it dips deep into the bulk, its time dependence is mild with $t^{\prime 2} \ll 1$ everywhere. This then shows that $t_{*}>t_{0}$ for $\frac{t_{0}}{r_{*}} \sim 1$ : numerically it can be checked that for $\frac{r_{*}}{t_{0}} \lesssim 3$ the $t(r)$ series above continues to satisfy $t_{*}>t_{0}$, i.e. the extremal surface bends in the direction away from the singularity. As $\frac{r_{*}}{t_{0}}$ increases further (keeping the limit $(3.22), t_{0}$ becomes smaller and it can be seen numerically that the series above violates $t_{*}>t_{0}$ : however in this regime, it can also be seen that $t^{\prime}$ is increasing so the analysis is breaking down: this occurs as we move the anchoring surface in the direction of the singularity, which becomes unreliable (not surprisingly).

The conditions (3.15), (3.16), on the local geometry in the neighbourhood of the turning point do not depend on $A$, so in particular they also apply in this IR limit. This is consistent with the spacelike condition being preserved here for generic strip size. Overall we see that such classical RT/HRT extremal surfaces exist for generic strip size. It is interesting that such a power series analysis works, since (3.17) and its $A=0$ limit are still complicated nonlinear equations: as we depart from the large $t_{0}$ semiclassical regime and move the anchoring surface toward the singularity, it is unclear if this can be analysed meaningfully. We have mainly analysed the AdS Kasner spacetime here for the time behaviour: however the techniques should be applicable to more general cosmologies of the kind described earlier.

The IR limit of large size $l$ can be probed in greater detail by quantum extremal surfaces as we will see in the next section. The findings there are consistent with the classical RT/HRT analysis here, but constrain $t_{*}, r_{*}$ further, owing to the bulk matter entropy contribution.

Finally, we now make some general statements based on energy conditions. Firstly for (3.14) as well as (3.9) and (3.10) to be well-behaved, we have seen that $\left(\partial_{r} t\right)^{2}<1$ which follows from requiring reality, and also $\phi \geq \phi_{*}=A$. The first condition is expected intuitively as we have seen. The second condition implies $\phi=\frac{t}{r^{d_{i}}}$ decreases till it becomes $\phi_{*}$, i.e.

$$
\phi \geq \phi_{*} \quad \rightarrow \quad \frac{t(r)}{r^{d_{i}}} \geq \frac{t_{*}}{r_{*}^{2 d_{i}}} \quad \rightarrow \quad \partial_{r}\left(\frac{t(r)}{r^{d_{i}}}\right) \leq 0 \quad \Rightarrow \quad \partial_{r} t \leq \frac{d_{i} t}{r} .
$$

The derivative condition follows from assuming monotonicity (with $r=0$ the boundary). This constrains the behaviour of the $t(r)$ function. More generally, this is reminiscent of null energy conditions, and the dilatonic c-function in [37]. Using the equations (2.3) in the 2-dim background, the NEC gives $-n^{\mu} n^{\nu} \nabla_{\mu} \nabla_{\nu} \phi=g^{t t} \nabla_{t} \nabla_{t} \phi-g^{r r} \nabla_{r} \nabla_{r} \phi \geq 0$, i.e. $-e^{f}\left(\partial_{t}^{2} \phi-\partial_{t} f \partial_{t} \phi+\partial_{r}^{2} \phi-\partial_{r} f \partial_{r} \phi\right) \geq 0$ which simplifies to $-\left(\phi^{\prime \prime}-f^{\prime} \phi^{\prime}-\frac{\left(d_{i}-1\right) / d_{i}}{t r^{d_{i}}}\right) \geq 0$ using (2.9). Restricting to the extremal surface we have $\phi=\frac{t(r)}{r^{d_{i}}}$ and $f=\frac{d_{i}-1}{d_{i}} \log t(r)-$ 
$\left(d_{i}+1\right) \log r$ which gives $t^{\prime \prime} \leq \frac{d_{i}-1}{d_{i}} \frac{1+t^{\prime 2}}{t}$. These general conditions appear consistent with the earlier discussions: although we have not used these much these considerations may provide interesting information in general cosmologies.

\section{Quantum extremal surfaces}

We will now study quantum extremal surfaces (QES) in the 2-dim cosmologies in [25] obtained by dimensional reduction from various higher dimensional theories, towards understanding the cosmological, Big-Crunch, singularities present here, in part inspired by the exciting findings in $[1,2]$. The relevant 2-dim fields here are the dilaton $\phi$, the 2-dim metric $e^{f}$ and the extra scalar $\Psi$. The scalar $\Psi$ is essential for nontrivial dynamics and essentially drives the singularity: however since the spacetime already contains the effects of the scalar we will assume that the scalar excitations are on the same footing as other bulk matter. This is equivalent to assuming that the effects of $\Psi$ have been subsumed into their backreaction on the geometry so using the background spacetime is adequate. Thus the bulk matter entropy $S_{\text {bulk }}$ will be assumed to contain contributions from the scalar $\Psi$ as well, which will not be treated separately. Towards putting this on firmer footing, it is important to understand the bulk entanglement entropy for scalars such as $\Psi$ with a dilaton coupling in the action (2.2): we will leave this for the future.

Consider an observer $O$ at some location $\left(t_{0}, r_{0}\right)$ moving in time in the spacetime background. In the time-dependent case, if he/she is far away from regions such as the Big-Crunch singularity, the time dependence is slow and it is reasonable to imagine that the ambient matter in the observer's neighbouring patch is in its ground state. Now we ask what entanglement he/she sees: say the QES is at some location $(t, r)$. We will use $\left(t_{*}, r_{*}\right)$ to refer to the QES solution to extremization of the generalized entropy,

$$
\begin{aligned}
S_{\text {gen }} & =\frac{\phi}{4 G_{2}}+S_{\text {bulk }} \\
& =\frac{\phi}{4 G_{2}}+\frac{c}{12} \log \left(\left.\Delta^{2} e^{f}\right|_{(t, r)}\right)+\ldots
\end{aligned}
$$

This is the classical area (dilaton) piece along with the subleading entropy of bulk matter. $\Delta^{2}$ is the flat spacetime interval between the observer $O$ and the QES,

$$
\Delta^{2}=r^{2}-\left(t-t_{0}\right)^{2}
$$

We focus on the observer $O$ located at the boundary $r=0$ since all the backgrounds we discuss have a holographic dual interpretation. We have written the expression (4.1) along the lines of the discussions in [2]: in particular the effects of the curved spacetime appear entirely through the conformal factor $e^{f}$ at the QES endpoint of the interval (see appendix $\mathrm{B}$ for a very brief recap). Above, we have only explicitly retained terms that are relevant for the QES extremization. So we have omitted terms containing the ultraviolet cutoffs and the warp factor $\left.e^{f}\right|_{t_{c}, r_{c}}$ at the boundary: the latter can be partially absorbed into the UV cutoffs. A useful resource for these QES calculations in time-independent cases is [38]. 
Several comments are in order on the generalized entropy (4.1) in our description and use: while some of these are also features of previous applications of the generalized entropy, some are specific to our context as will be clear in what follows.

1. The only boundary subregions that make sense in these reduced 2-dim bulk theories are the whole space from the higher dimensional point of view. So the leading term is the transverse area of the full space in the higher dimensional theory, which is the 2-dim dilaton (in Planck units). From the expression (3.2) for the RT/HRT surface in the higher dimensional theory, this leading classical term can be seen to arise as

$$
S_{\text {gen }}^{c l} \sim \frac{V_{d_{i}-1}}{4 G_{d_{i}+2}} \int d r \phi \sqrt{\left(\partial_{r} x\right)^{2}} \sim \frac{V_{d_{i}-1}}{4 G_{d_{i}+2}} \int d x \phi \sim \frac{\phi}{4 G_{2}} .
$$

using $\frac{1}{G_{2}} \sim \frac{V_{d_{i}}}{G_{d_{i}+2}}$. In the 2-dim theory, the extremal surface is just a point in the 2dim spacetime, the entire transverse part of the higher dim extremal surface wrapped. The QES location $\left(t_{*}, r_{*}\right)$ after extremization of the generalized entropy (4.1) is roughly speaking analogous to the turning point in the classical RT/HRT analysis, and the 2-dim discussion pertains to the IR limit there.

In time-independent situations, the boundary subregion can be taken to be a point on the boundary $r=r_{c}$ on some constant time slice, and then the extremal surface is a point lying at some spatial location $r=r_{*}$ on that slice. We will describe some examples of this below.

2. In general we will assume that the bulk matter is a 2-dim conformal field theory. Then the bulk entanglement entropy [5,6] of quantum matter fields in the bulk subregion enclosed by the extremal surface and the boundary subregion can be described by the Calabrese-Cardy expression [39, 40] for a 2-dim CFT in flat space, along with the modifications from the conformal transformation to the curved 2-dim space [2]. The interval has endpoints defined by the extremal surface at $(t, r)$ and the boundary $\left(t_{0}, r_{c}\right) \sim\left(t_{0}, 0\right)$ : thus $S_{\text {bulk }}$ has been obtained using the rules of boundary CFT, with a single twist operator at the QES endpoint (some details appear in appendix B). For the cosmological spacetimes, nontrivial time dependence arises from the interval but mainly from the conformal transformation, which as we will see is nontrivial.

We have also assumed in writing this expression that

$$
1 \ll c \ll \frac{1}{G},
$$

i.e. the matter CFT central charge is sufficiently large to give nontrivial subleading contributions to the generalized entropy, but not too large that it backreacts and wrecks the classical geometry (and thereby the classical entanglement term).

In the higher dimensional theory, the bulk entropy contribution $[5,6]$ is in general difficult to calculate: we resort to the effective 2-dim theories where $S_{\text {bulk }}$ can be approximated by 2-dim CFT entanglement entropy. 
3. In writing $S_{\text {bulk }}$ we have assumed that the quantum matter fields are in a pure state, and for simplicity we have assumed the ground state of the CFT for most of our analysis. This is reasonable if the background is time-independent, or has slow time variation. In the cosmological cases we discuss, this form of $S_{\text {bulk }}$ is reasonable in the semiclassical spacetime region far from the singularity where the time variations are not significant. Since the warp factor $e^{f}$ contains time-dependence, $S_{\text {bulk }}$ contains effects of time evolution: we expect this to be reasonable for mild time dependence.

However in the cosmological spacetimes we discuss, there are global Big-Crunch singularities where the entire spacetime becomes vanishingly small with the conformal factor $e^{f}$ going to zero: this suggests a singularity arising from $\log e^{f}$. Physically one might imagine that the severe time-dependence would lead to the bulk matter going to some excited state, perhaps severely excited near the singularity. This suggests a breakdown of $S_{\text {bulk }}$, and in fact the entire semiclassical approach in these techniques which are unceremoniously being extrapolated to a region with large quantum gravity effects. As it turns out, our analysis appears self-consistent in the sense that the quantum extremal surfaces end up being localized in the semiclassical spacetime region far from the singularity. We will comment on these further after we discuss the analysis.

\subsection{Some time-independent backgrounds}

Before studying the cosmological backgrounds, we will first study some time-independent backgrounds to gain some intuition and experience for the above generalized entropy (4.1) and the resulting quantum extremal surfaces. With no time-dependence, all time slices are equivalent so we can set $t=t_{0}$, i.e. the QES lies on the same time slice as the observer. This is of course borne out in our experience with entangling surfaces in time-independent backgrounds in higher dimensional holography.

$\boldsymbol{A d} \boldsymbol{S}_{\mathbf{2}}$. Here we have $d s^{2}=\frac{1}{r^{2}}\left(-d t^{2}+d r^{2}\right)$ with $\phi=\frac{\phi_{r}}{r}$ and the generalized entropy (4.1) setting $t=t_{0}$ becomes

$$
S_{\text {gen }}=\frac{\phi_{r}}{4 G} \frac{1}{r}+\frac{c}{6} \log \left(r \frac{1}{r}\right) ; \quad \partial_{r} S_{\text {gen }} \sim-\frac{\phi_{r}}{r^{2}} \rightarrow 0 .
$$

We have retained only terms relevant for the extremization. We see that the warp factor at the $r$-endpoint cancels the $r$-dependence of the interval entanglement, giving just the classical piece. Thus the extremization gives the second expression above so the solution to extremization is $r_{*} \rightarrow \infty$. Thus the entanglement wedge [41-43] defined as the bulk domain of dependence of the QES (the part of the spacetime causally connected to the QES at $\left.r_{*} \rightarrow \infty\right)$ is the entire Poincare wedge as expected. See also [38] for discussions on this.

$\boldsymbol{A d} \boldsymbol{S}_{\boldsymbol{D}}$ reduction. The higher $\operatorname{dim} A d S_{D}$ space with $D=d_{i}+2$ is $d s_{A d S_{d_{i}+2}}^{2}=\frac{1}{r^{2}}\left(-d t^{2}+\right.$ $\left.d r^{2}\right)+\frac{1}{r^{2}} d x_{i}^{2}$ and under reduction (2.1) we obtain the 2-dim background (suppressing the AdS scale)

$$
\phi=\frac{1}{r^{d_{i}}}, \quad d s^{2}=\frac{1}{r^{d_{i}+1}}\left(-d t^{2}+d r^{2}\right)
$$


Some aspects of such generic 2-dim dilaton gravity theories have been discussed in [32]. Now (4.1) gives

$$
S_{\text {gen }}=\frac{\phi_{r}}{4 G} \frac{1}{r^{d_{i}}}+\frac{c}{6} \log \left(\frac{r}{r^{\left(d_{i}+1\right) / 2}}\right) \quad \Rightarrow \quad \partial_{r} S_{\text {gen }}=-\frac{d_{i} \phi_{r}}{4 G r^{d_{i}+1}}-\frac{c}{6}\left(\frac{d_{i}-1}{2}\right) \frac{1}{r}=0 .
$$

We see that both terms are of the same sign since $c>0$ and $d_{i}>1$. Thus the solution is again $r_{*} \rightarrow \infty$ for the location of the QES: this again leads to the entire Poincare wedge which is the expected answer (also in the higher dimensional point $A d S_{D}$ when the subsystem becomes the whole space). Note that we are using the 2-dim metric as the Weyl transformed one (2.1) in (4.6) above: this was found to be consistent in [32] in holographic discussions (e.g. the stress tensor).

It is to be noted that we have written $S_{\text {bulk }}$ using the rules of boundary CFT since the effective space is the half-line with one end of the interval at the boundary $r=0$. It is instructive to compare this with the discussion of islands in e.g. [44], where a flat region was appended beyond the boundary $r=0$ of an $A d S_{2}$ region: in this case, the generalized entropy takes the form $S_{\text {gen }} \sim \frac{\phi_{r}}{4 G} \frac{1}{r}+\frac{c}{6} \log \left(\left(r+r^{\prime}\right)^{2} \frac{1}{r}\right)$. The interval in question has endpoints $r \in A d S_{2}$ and $r^{\prime}$ in the flat space region beyond the boundary: the warp factor at the $r^{\prime}$ end does not contribute since it is trivial in that flat region. Both $r, r^{\prime}>0$ in this parametrization: the space is not a half-line now. Let us set $r^{\prime} \sim 0$ for simplicity. Then extremizing gives $-\frac{\phi_{r}}{4 G} \frac{1}{r^{2}}+\frac{c}{6} \frac{1}{r}=0$ : the competition between the two terms leads to a finite value $r_{*} \sim \frac{\phi_{r}}{G c}$ for the QES location. In the case (4.7) above we see that the argument of the logarithm in the bulk entropy arises differently and $r_{*} \rightarrow \infty$ with no island.

One way to understand this is in terms of the violation of the Bekenstein bound, as discussed in [13]: if the classical dilatonic term is overpowered by the subleading bulk entropy contribution, we may expect islands. To see this, note that (4.7) can be recast as

$$
S_{\mathrm{gen}}=\frac{\phi}{4 G}+\frac{c}{6} \frac{d_{i}-1}{d_{i}} \log \phi
$$

with a relative plus sign in the two contributions, again retaining only terms relevant for extremization (in greater detail, putting the AdS scale and the UV cutoff scales back, the bulk entropy term is $\log \left(\phi^{\left(d_{i}-1\right) / d_{i}} \frac{R^{2}}{\epsilon_{1} \epsilon_{2}}\right)$ : the argument becomes $O(1)$ when $\phi$ is sufficiently small, at large $r$ ). As long as $\phi$ is not too small, $\log \phi$ will always be subdominant to the classical area term $\phi$ and the Bekenstein bound will not be violated: thus the extremization leads to $\partial_{r} \phi=0$ giving $r_{*} \rightarrow \infty$ which is the entire Poincare wedge, with no islands. If one could somehow entangle the bulk matter on the interval with some other region, this may lead to $S_{\text {bulk }}$ increasing and overpowering the classical area contribution: this is what appears to be happening in the example above from [44], as well as various cases discussed in [13].

\subsection{2-dim cosmologies and quantum extremal surfaces}

Now we will study quantum extremal surfaces in the 2-dim cosmological backgrounds reviewed earlier. We focus first on the 2-dim cosmology obtained by reduction of the $A d S_{D}$ Kasner spacetime (2.9). With the observer at the boundary and the interval $\Delta^{2}$ 


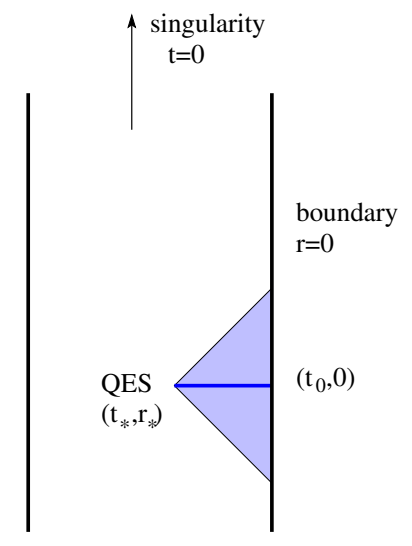

Figure 3. Cartoon of the 2-dim geometry, the holographic boundary at $r=0$ and the QES at $\left(t_{*}, r_{*}\right)$. The solid blue line is the spatial interval between the boundary observer and the QES. The singularity is at $t=0$ far up (not shown). The extremization drives the QES location to $t_{*} \rightarrow \infty$ (far from the singularity) and $r_{*} \rightarrow \infty$ (which is the left vertical line).

between the observer and the QES location as in (4.2), we obtain

$$
\begin{aligned}
S_{\text {gen }} & =\frac{\phi_{r}}{4 G} \frac{t}{r^{d_{i}}}+\frac{c}{12} \log \left[\left.\left(r^{2}-\left(t-t_{0}\right)^{2}\right) e^{f}\right|_{(t, r)}\right]+\ldots \\
\partial_{r} S_{\text {gen }} & =-\frac{\phi_{r}}{4 G} \frac{d_{i} t}{r^{d_{i}+1}}+\frac{c}{6} \frac{r}{r^{2}-\left(t-t_{0}\right)^{2}}-\frac{c}{12} \frac{d_{i}+1}{r}=0, \\
\partial_{t} S_{\text {gen }} & =\frac{\phi_{r}}{4 G} \frac{1}{r^{d_{i}}}-\frac{c}{6} \frac{t-t_{0}}{r^{2}-\left(t-t_{0}\right)^{2}}+\frac{c}{12} \frac{d_{i}-1}{d_{i} t}=0 .
\end{aligned}
$$

We are again only retaining terms relevant for extremization. Some general comments can be made here: assuming as is usually the case in $A d S / C F T$, the entanglement wedge lies outside the causal wedge (deeper in the interior) [41-43], we have $\Delta^{2}>0$. For the usual parametrization of the bulk, we have $r>0$ : this also implies $\Delta^{2}>0$ for a real solution (independent of the argument above). Rearranging (4.10) gives

$$
\frac{c}{6} \frac{r}{r^{2}-\left(t-t_{0}\right)^{2}}=\frac{\phi_{r}}{4 G} \frac{d_{i} t}{r^{d_{i}+1}}+\frac{c}{12} \frac{d_{i}+1}{r}, \quad \frac{c}{6} \frac{t-t_{0}}{r^{2}-\left(t-t_{0}\right)^{2}}=\frac{\phi_{r}}{4 G} \frac{1}{r^{d_{i}}}+\frac{c}{12} \frac{d_{i}-1}{d_{i} t} .
$$

Since $d_{i}>1$, the right side is positive always, so we have

$$
\Delta^{2}>0 \text { i.e. }\left(t-t_{0}\right)^{2}<r^{2}, \quad r>0, \quad t \geq t_{0} .
$$

$t \geq t_{0}>0$ means the QES lies on the same time slice as or beyond the observer, further away from the singularity.

If we assume that the QES lies on the same time slice as the observer i.e. $t=t_{0}$, equivalently that the QES is maximally spacelike separated from the observer, we obtain from (4.9)

$$
\begin{aligned}
t & =t_{0}: \quad S_{\text {gen }}=\frac{\phi}{4 G}+\frac{c}{6} \frac{d_{i}-1}{d_{i}} \log \phi, \quad \phi & =\frac{\phi_{r}}{4 G} \frac{t}{r^{d_{i}}}, \\
\partial_{r} S_{\text {gen }} & \sim-\left(\frac{\phi_{r}}{4 G} \frac{d_{i} t}{r^{d_{i}+1}}+\frac{c}{12} \frac{d_{i}-1}{r}\right)=0, \quad \partial_{t} S_{\text {gen }} & \sim \frac{\phi_{r}}{4 G} \frac{1}{r^{d_{i}}}+\frac{c}{12} \frac{d_{i}-1}{d_{i} t}=0 .
\end{aligned}
$$


Since $c>0$ and $d_{i}>1$, both contributions in both derivative expressions appear with the same sign. Note also that in this entire discussion, as described after (3.11), we are on one side (the past) of the singularity at $t=0$, so the range of the time variable is $t \equiv|t| \geq 0$. Then it is clear that the only solution $\left(t_{*}, r_{*}\right)$ to extremization is

$$
r \equiv r_{*} \rightarrow \infty, \quad t \equiv t_{*} \rightarrow \infty ; \quad t_{*} \lesssim r_{*} .
$$

This condition is somewhat analogous to (3.22). Since we have assumed $t=t_{0}$, this is consistent only if $t_{0} \rightarrow \infty$ also, i.e. the observer lies far from the singularity in the semiclassical spacetime region. It is useful to note that the first expression $\partial_{r} S_{\text {gen }}$ can be regarded as a spatial minimization on a fixed Cauchy $t$-slice, which is solved by $r_{*} \rightarrow \infty$ for any $t$-value (not growing faster than $r$ ): we see that $\partial_{r}^{2} S_{\text {gen }}>0^{+}$. Then the second expression $\partial_{t} S_{\text {gen }}$, which is a maximization, forces $t_{*} \rightarrow \infty$ : we have $\partial_{t}^{2} S_{\text {gen }} \sim-\frac{c}{t^{2}} \rightarrow 0^{+}$. It is also interesting to note from (4.13) that

$$
\partial_{r} S_{\text {gen }} \sim-\frac{d_{i} t}{r} \partial_{t} S_{\text {gen }} .
$$

This is a nontrivial relation, which is true only because the $t$ - and $r$-exponents in $\phi, e^{f}$ are related in a nontrivial manner. This also explains the last condition on $t_{*}, r_{*}$ in (4.14): if this were violated, then the extremization could be violated particularly in $\partial_{r} S_{\text {gen }}$. Roughly this is consistent with preserving the spacelike condition in some sense, in particular (4.12). We will expand further on the relation (4.15) when we discuss more general cosmologies later.

In the form (4.13) for $S_{\text {gen }}$, it is clear that there is no Bekenstein bound violating region since the $S_{\text {bulk }}$ term is always subleading to the classical area term as long as $\phi$ is not small. Thus the spatial extremization in $r$ is expected to lead to $\phi \rightarrow 0$ or $r_{*} \rightarrow \infty$. However it is instructive to note that the $t$-extremization can be written as

$$
\frac{\partial_{t} \phi}{4 G}+\frac{c}{6} \frac{d_{i}-1}{d_{i}} \frac{\partial_{t} \phi}{\phi}=0
$$

The fact that $\phi \sim \frac{t}{r^{d_{i}}}$ with the Big-Crunch singularity at $t=0$ and noting that both terms here contribute with the same sign implies that the only solution to extremization is at $r_{*} \rightarrow \infty$ from the first term and $t_{*} \rightarrow \infty$ from the second. These automatically solve the $r$-extremization as well, as long as $t_{*} \lesssim r_{*}$. Another way to see this condition arising is to note that $S_{\text {bulk }}$ positivity implies $\phi$ is not too small. To see this in more detail, let us reinstate lengthscales back: these are the AdS scale $R$ and the Kasner scale $t_{K}$ implicit in the $A d S_{D}$ Kasner spacetime (2.9), although $t_{K}$ does not play a crucial role. This gives

$$
S_{\text {bulk }} \sim c \log \left(\frac{r^{2}}{\epsilon_{1} \epsilon_{2}} \frac{\left(t / t_{K}\right)^{\frac{d_{i}-1}{d_{i}}} R^{d_{i}+1}}{r^{d_{i}+1}}\right) \sim c \log \left(\phi^{\frac{d_{i}-1}{d_{i}}} \frac{R^{2}}{\epsilon_{1} \epsilon_{2}}\right)
$$

Thus $S_{\text {bulk }}$ becomes negative as $\phi \rightarrow 0$, strictly when $\phi$ becomes smaller than $\frac{R^{2}}{\epsilon_{1} \epsilon_{2}}$. From the higher dimensional point of view, the area of the transverse space must become sufficiently small in units of the AdS scale and the ultraviolet cutoff scales. It is simplest to interpret this as a breakdown of these expressions in the near singularity region. 
It is also interesting to compare this analysis of the generalized entropy with the classical area term extremization: we have

$$
\partial_{r} \phi=-\frac{t}{r^{d_{i}+1}}=0, \quad \partial_{t} \phi=\frac{1}{r^{d_{i}}}=0
$$

both of which are solved by $r_{*} \rightarrow \infty$, as long as $t_{*} \lesssim r_{*}$. This classical area term does not force the extremal surface to be driven to $t_{*} \rightarrow \infty$ : in particular we see that $t_{*}=0$ also appears formally consistent with these classical extremization equations. However this entire formulation is unreliable in the vicinity of $t=0$. The situation is perhaps best appreciated in light of the higher dimensional RT/HRT analysis earlier: from (3.8), (3.11), we see that

$$
\phi_{*}=\frac{t_{*}}{r_{*}^{d_{i}}} \sim \frac{1}{l^{d_{i}}} \rightarrow 0
$$

in the IR limit $l \rightarrow \infty$ (see the discussion towards the end of section3) which is the regime probed by the quantum extremal surface in this 2-dimensional analysis here. From the higher dimensional perspective, the surface dips deep into the bulk so $r_{*}$ increases: but in the semiclassical regime as we saw analysing (3.17), the dip in time is mild, with $t_{*} \gtrsim t_{0}$.

Looking more closely, we see that what necessitates driving the quantum extremal surface to $t_{*} \rightarrow \infty$ is the $\frac{1}{t}$ term in (4.11), (4.13). This stems from the power-law BigCrunch $e^{f} \sim t^{\#}$ factor in (4.1) which gives a $\log t$ term. If there is no complete Big-Crunch (for instance as in a bouncing $\operatorname{cosmolog} y^{1}$ ), such a term would perhaps not arise, allowing finite $t_{*}$ values.

So far we have been discussing this taking the quantum extremal surface to be maximally spacelike separated from the observer so $t=t_{0}$. This is consistent, as we have seen, with the observer located far from the singularity since the QES is located far from the singularity. Now going back to the more general case (4.11), it is instructive to note the following. The first equation in (4.11) is satisfied for $r=R_{c} \sim \infty$, regulating $r_{*} \rightarrow \infty$ to $r_{*} \sim R_{c}$. Then the second equation can be approximated as

$$
\frac{t-t_{0}}{R_{c}^{2}} \sim \frac{d_{i}-1}{2 d_{i}} \frac{1}{t} \Rightarrow t_{*} \sim \frac{t_{0}+\sqrt{t_{0}^{2}+4 A R_{c}^{2}}}{2}
$$

which shows $t_{*} \sim R_{c}$ as the QES location in (4.11) regulated from infinity to $R_{c} \gg 1$. This arises entirely from the $c$-dependent quantum (bulk) entanglement term.

Now consider the case where the observer is very close to the singularity, i.e. $t_{0} \sim 0$ : this is a bad approximation and we expect a breakdown but it is instructive to analyse this. Then (4.11) with $t_{0} \sim 0$ become

$$
\frac{c}{6} \frac{r}{r^{2}-t^{2}} \sim \frac{\phi_{r}}{4 G} \frac{d_{i} t}{r^{d_{i}+1}}+\frac{c}{12} \frac{d_{i}+1}{r}, \quad \frac{c}{6} \frac{t}{r^{2}-t^{2}} \sim \frac{\phi_{r}}{4 G} \frac{1}{r^{d_{i}}}+\frac{c}{12} \frac{d_{i}-1}{d_{i} t} .
$$

\footnotetext{
${ }^{1}$ For instance a warp factor $e^{f} \sim\left(t^{2}+\delta\right)^{\frac{d_{i}-1}{2 d_{i}}}$ exhibits a bounce at $t=0$ without crunching to zero entirely, $\delta$ being a regulator. However usually bounces of this kind require violating energy conditions or other nonstandard physics. In any case with this form of $e^{f}$ it appears that $t=0$ is also a solution to the extremization: it would seem that this is very unreliable since we expect severe quantum gravity effects here.
} 
One might wonder if the QES lies in the vicinity of the singularity also, i.e. $t=\delta \sim 0$. The first equation is satisfied if $r_{*} \rightarrow \infty$ but the second is not satisfied due to the last term which diverges as $\delta \rightarrow 0$. This last term suggests that in fact the QES perhaps lies at $t_{*} \rightarrow \infty$ again. Rather than attempting to look for exact solutions, we will look for a scaling solution: this will point to the QES again lying far from $t=0$. Since $\Delta^{2}=r^{2}-t^{2}>0$, consider a trajectory $r=\lambda t$ as an ansatz for identifying if the QES lies at infinity or at zero (near the singularity): this is consistent since for both infinity and zero, the scaling ansatz might be expected to confirm or rule out if the QES lies at zero, i.e. in the vicinity of the singularity. This gives

$$
\frac{c}{12}\left(\frac{2 \lambda}{\lambda^{2}-1}-\frac{d_{i}+1}{\lambda}\right) \frac{1}{t}=\frac{\phi_{r}}{4 G \lambda^{d_{i}+1}} \frac{d_{i}}{t^{d_{i}}}, \quad \frac{c}{12}\left(\frac{2}{\lambda^{2}-1}-\frac{d_{i}-1}{d_{i}}\right) \frac{1}{t}=\frac{\phi_{r}}{4 G \lambda^{d_{i}}} \frac{1}{t^{d_{i}}} .
$$

In general, the coefficients of the $\frac{1}{t}$ and $\frac{1}{t^{d_{i}}}$ terms are different: ${ }^{2}$ so each term must vanish independently: thus the only solution is $t_{*} \rightarrow \infty$ and thereby $r_{*} \rightarrow \infty$.

Thus this formulation of the generalized entropy appears to self-consistently exclude the near singularity region. In a sense the fact that the quantum extremal surfaces are driven far from the singular region is reassuring with regard to the Page curve findings [1-3] which appear to not require any information from the near singularity region.

\subsection{More general 2-dim cosmologies}

We now make a few comments on the generalized entropy and quantum extremal surfaces in more general 2-dim cosmologies with the general scaling form (2.5), defined by various $t$ and $r$-exponents used in [25]. We will also assume for simplicity that the QES is maximally spacelike separated from the observer, thus setting $t=t_{0}$ in the general expression (4.1). Finally since all these cosmologies have a boundary at $r=0$ we will restrict attention to such boundary observers. This gives

$$
S_{\text {gen }}=\frac{\phi}{4 G}+\frac{c}{12} \log \left(\left.r^{2} e^{f}\right|_{(t, r)}\right)=\frac{t r^{m}}{4 G}+\frac{c}{12} \log \left(t^{a} r^{b+2}\right)
$$

retaining only terms relevant for the extremization as before. From the various examples in section 2 , we know that $m, b<0$ : this is in accord with the transverse space, i.e. dilaton, expanding towards the boundary $r \sim 0$. Further we have also used the universality of the time exponent of the dilaton. Firstly, this can be recast in the schematic form (4.13) only if the argument of the logarithm is related to the dilaton exponents appropriately: this gives

$$
S_{\mathrm{gen}} \equiv \frac{\phi}{4 G}+\frac{c a}{12} \log \phi \quad \Leftrightarrow \quad a=\frac{2+b}{m} .
$$

\footnotetext{
${ }^{2}$ For instance, as we "dial" $\lambda$ from $\lambda \gg 1$ to $\lambda=1+\varepsilon$, we have

$\frac{c}{12} \frac{1-d_{i}}{\lambda} \frac{1}{t} \sim \frac{\phi_{r}}{4 G \lambda^{d_{i}+1}} \frac{d_{i}}{t^{d_{i}}}, \quad \frac{c}{12} \frac{1-d_{i}}{d_{i}} \frac{1}{t} \sim \frac{\phi_{r}}{4 G \lambda^{d_{i}}} \frac{1}{t^{d_{i}}} ; \quad \frac{c}{12} \frac{1}{\varepsilon} \frac{1}{t} \sim \frac{\phi_{r}}{4 G} \frac{d_{i}}{t^{d_{i}}}, \quad \frac{c}{12} \frac{1}{\varepsilon} \frac{1}{t} \sim \frac{\phi_{r}}{4 G} \frac{1}{t^{d_{i}}}$, vindicating the $t_{*} \rightarrow \infty$ solution.
} 
It is interesting to note that this relates the $t$ - and $r$-exponents which were otherwise independent in general in [25]. Extremization of (4.23) gives

$$
\begin{aligned}
& \partial_{r} S_{\text {gen }}=\frac{m t r^{m-1}}{4 G}+\frac{c}{12} \frac{2+b}{r}=0, \quad \partial_{t} S_{\text {gen }}=\frac{r^{m}}{4 G}+\frac{c}{12} \frac{a}{t}=0, \\
& \Rightarrow \quad \partial_{r} S_{\text {gen }} \sim \frac{m t}{r} \partial_{t} S_{\text {gen }} \quad \Leftrightarrow \quad a=\frac{2+b}{m} .
\end{aligned}
$$

From the time extremization $\partial_{t} S_{\text {gen }}=0$ and noting $m<0$ we see that the solution to the QES location is $r_{*} \rightarrow \infty, t_{*} \rightarrow \infty$, with $t_{*} \lesssim r_{*}$. This structure is similar to (4.14) for the 2-dim cosmology obtained from the AdS Kasner reduction that we discussed earlier. Now looking at just the classical area term, with a scaling form $\phi=t^{k} r^{m}$, extremizing gives

$$
\partial_{r} \phi=m t^{k} r^{m-1}=0, \quad \partial_{t} \phi=k t^{k-1} r^{m}=0 .
$$

Since the dilaton grows towards the boundary $r \rightarrow 0$, we must have $m<0$. Then for $k>0$, these equations are similar to (4.18), with both satisfied if $r_{*} \rightarrow \infty$ as long as $t_{*} \lesssim r_{*}$. With the universality (2.7), taking $k=1$ so $\phi=t r^{m}$, a general bulk matter entropy $S_{\text {bulk }}$ gives

$$
S_{\mathrm{gen}}=\frac{t r^{m}}{4 G_{2}}+S_{b} ; \quad \partial_{r} S_{\mathrm{gen}}=\frac{m t r^{m-1}}{4 G_{2}}+\partial_{r} S_{b}=0, \quad \partial_{t} S_{\mathrm{gen}}=\frac{r^{m}}{4 G_{2}}+\partial_{t} S_{b}=0 .
$$

For $S_{b}$ being the ground state entanglement, we obtain (4.25), (4.26) above, for the scaling form. More generally, a relation of the form (4.26) arises for $S_{\text {gen }}$ if $S_{\text {bulk }}$ satisfies $\partial_{r} S_{b}=\frac{m t}{r} \partial_{t} S_{b}$. For instance an extensive bulk entropy $S_{\text {bulk }}$ of the form below gives

$$
\begin{aligned}
S_{\mathrm{bulk}} & =\left.\Lambda r e^{f / 2}\right|_{(t, r)}=\Lambda t^{a / 2} r^{(2+b) / 2} \rightarrow \\
\partial_{r} S_{\mathrm{gen}} & =\frac{m t r^{m-1}}{4 G_{2}}+\Lambda t^{a / 2} r^{b / 2}=0, \quad \partial_{t} S_{\text {gen }}=\frac{r^{m}}{4 G_{2}}+\Lambda t^{(a-2) / 2} r^{(2+b) / 2}=0,
\end{aligned}
$$

thus satisfying the relation (4.26) if $a=\frac{2+b}{m}$. In particular this is true for the AdS Kasner reduction earlier. However this generalized entropy can be seen to vanish at the location $t=0$ of the singularity: it is unclear if this is reasonable. It would be interesting to explore good models for near singularity physics and the resulting quantum extremal surfaces.

\section{Discussion}

We have studied aspects of entanglement and extremal surfaces in various families of spacetimes exhibiting cosmological, Big-Crunch, singularities, in particular the isotropic AdS Kasner spacetime. The classical RT/HRT extremal surface dips into the bulk radial and time directions, with turning points (3.8), (3.11), satisfying $l \sim r_{*}$ and $\phi_{*}=A=\frac{t_{*}}{r_{*}}$, for $A d S_{d_{i}+2}$ Kasner. By analysing the time extremization equation in the reliable semiclassical region far from the singularity via (3.17), we have seen explicitly that the surface lies mostly on a constant time slice and bends in the direction away from the singularity at $t=0$. At the turning point, the surface exhibits time-maximization. As we have seen, the IR limit where $A \rightarrow 0$ continues to exhibit such behaviour, but also shows indications of 
the analysis breaking down. In the 2-dim cosmologies obtained by dimensional reduction of these and other singularities [25], we have studied quantum extremal surfaces. The generalized entropy (4.1) comprises the classical area (dilaton) term and a bulk matter entropy obtained by using the formulation of $[1,2]$ with the effects of the curved space incorporated via the conformal transformation, taking the matter in the ground state in flat space (in the region far from the singularity at $t=0$ ). The resulting extremization shows the quantum extremal surfaces exhibit a maximin structure: they are always driven to the semiclassical region far from the singularity (e.g. (4.9), (4.13) for the isotropic AdS Kasner reduction). Technically this follows from the Crunching term in the warp factor. We do not find islands in this analysis: this appears consistent with previous investigations on closed universes, and can be interpreted in terms of the Bekenstein bound not being violated [13], so that there is no competition for the area (dilaton) term. It would be interesting to consider extra regions elsewhere (e.g. flat space regions beyond the boundary $r=0$ or in the far past) that are entangled with these universes: these may exhibit islands.

In the discussion of quantum extremal surfaces, we have used 2-dim CFT techniques: these are technically reasonable in the 2-dim theories assuming that the bulk matter is described by a CFT. These 2-dim backgrounds are consistent intrinsically as solutions to the 2-dim dilaton-gravity-scalar theories (2.2), so the formulation of generalized entropy here is consistent in the semiclassical regime. These would seem to faithfully capture qualitative features of quantum extremal surfaces in the higher dimensional cosmologies that give rise to the 2-dim backgrounds upon dimensional reduction, at least considering that the surfaces are driven to the semiclassical region. In some "effective holography" sense, the 2-dim backgrounds faithfully reflect the higher dimensional description (see [32] for more discussions in this regard). However it would seem that this would break down had the vicinity of the singularity entered: happily the quantum extremal surfaces avoid this.

In a sense these are reminiscent of similar features noted in the study of the HartmanMaldacena extremal surfaces [45] in the AdS black hole where the extremal surface approached a limiting surface some distance from the singularity in the interior (similar limiting surfaces were found in [46] in de Sitter: it would be interesting to understand quantum extremal surfaces in that context); similar observations were noted also in the AdS Kasner soliton [36], and other cases. We offer some comments and speculations on these results. Perhaps the simplest understanding hinges on the fact that the near singularity region is necessarily a place where quantum gravity effects are severe and the formulation of quantum extremal surfaces, such as it is, is simply not adequate. A more detailed model of the near singularity region incorporating perhaps "stringy entanglement" may be necessary. Our studies here seem consistent with the recent excitement on black holes and the Page curve: all the action there remained well separated from the near singularity region in the deep interior of the black hole (this singularity is anisotropic Kasner). In a sense the study here is reassuring since the quantum extremal surfaces self-consistently avoid the near singularity region rife with quantum gravity effects, remaining in semiclassical regimes far away. Turning this around, one might speculate if such Big-Crunch singularities are perhaps disallowed in string theory and holography, based on the (naive) diagnostic that entanglement via extremal surfaces is strictly incapable of probing the vicinity of such singularities. It 
is worth noting that the models we have studied pertain to AdS-cosmologies and related backgrounds (the universality (2.7) found in [25] suggests that the singularity nature of all such backgrounds is similar). These have a timelike boundary at $r=0$ with a holographic dual field theory interpretation: the extremal surfaces we have discussed thus encode entanglement observables in the dual field theories. It may be interesting to understand if more general cosmologies without such holographic restrictions exhibit similar features. It is also important to note that other holographic cosmologies may not exhibit this sort of repulsive behaviour: an example is anisotropic AdS Kasner with some directions expanding and some Crunching, but this lies outside the reduction to the 2-dim space we have employed.

Relatedly it may also be worth noting that perhaps assuming that bulk matter is in its ground state far from the singularity in our models is a nontrivial assumption: ${ }^{3}$ generic initial Cauchy data might be expected to lead to black hole formation rather than a Big-Crunch singularity (further discussions appear in [25]). From this point of view, it is perhaps not surprising that the generalized entropy incorporating ground state matter entanglement leads to quantum extremal surfaces that avoid the singularity. Perhaps a better model might incorporate more nontrivial initial conditions for the bulk matter and the associated entropy would then naturally lead to a Big-Crunch singularity that is accessible via entanglement. It would be interesting to explore these issues further.

\section{Acknowledgments}

It is a pleasure to thank Tom Hartman for a very useful early discussion, and Tom Hartman, Arnab Kundu and Raghu Mahajan for comments on a draft. We thank Ritabrata Bhattacharya for collaboration in the initial stages of this work, as well as Alok Laddha for discussions. This work is partially supported by a grant to CMI from the Infosys Foundation.

\section{A Further details on $t(r)$ for classical extremal surfaces}

We shall describe some relevant details of the behaviour of the extremal surface in the higher dimensional case. In the $A d S_{5}$ case, the $t(r)$ function, as a power series solution to (3.17), is

$$
\begin{aligned}
t(r)= & t_{0}+\frac{1}{12 t_{0}} r^{2}-\frac{1}{432 t_{0}^{3}} r^{4}+\frac{1}{7776 t_{0}^{5}} r^{6}+\frac{\left(-17+11664 A^{2} t_{0}^{4}\right)}{1866240 t_{0}^{7}} r^{8}+\frac{\left(247-400464 A^{2} t_{0}^{4}\right)}{335923200 t_{0}^{9}} r^{10} \\
& +\frac{\left(-1819+5110128 A^{2} t_{0}^{4}\right)}{28217548800 t_{0}^{11}} r^{12}+\frac{\left(21277-90004284 A^{2} t_{0}^{4}+5555329920 A^{4} t_{0}^{8}\right)}{3555411148800 t_{0}^{13}} r^{14} \\
& +\frac{\left(-373318170624 A^{4} t_{0}^{8}+2088752184 A^{2} t_{0}^{4}-355981\right)}{614375046512640 t_{0}^{15}} r^{16}
\end{aligned}
$$

\footnotetext{
${ }^{3}$ This is in certain cases consistent with a Euclidean continuation which might lead to a natural initial state (although generic time-dependent spacetimes become complex). For instance de Sitter space $d s^{2}=$ $\frac{R_{d S}^{2}}{\tau^{2}}\left(-d \tau^{2}+d x_{i}^{2}\right)$ under the analytic continuation $\tau \rightarrow i r, R_{d S} \rightarrow i R_{\mathrm{AdS}}$ becomes Euclidean AdS with corresponding continuations for fields and their boundary conditions; see also e.g. [15, 47] in other contexts. Then we see that (2.9) naively admits a similar Euclidean continuation $t \rightarrow i \tau, t_{K} \rightarrow i \tau_{K}$, if we also analytically continue the Kasner scale $t_{K}$ : equivalently, on the $t=t_{K}$ time slice sufficiently far from the singularity, we could consider gluing e.g. a flat region, in part along the lines of [12], which appears consistent with taking matter in the ground state. We hope to study this in greater detail in future work towards understanding initial conditions for spacetimes developing such Big-Crunch singularities.
} 


$$
\begin{aligned}
& +\frac{\left(257320977209856 A^{4} t_{0}^{8}-740910019032 A^{2} t_{0}^{4}+96110087\right)}{1658812625584128000 t_{0}^{17}} r^{18} \\
& +\frac{\left(707587260600729600 A^{6} t_{0}^{12}-35768655789931008 A^{4} t_{0}^{8}+63403487354664 A^{2} t_{0}^{4}-6506954915\right)}{1094816332885524480000 t_{0}^{19}} r^{20} \\
& +\ldots
\end{aligned}
$$

One can compute higher order coefficients iteratively using Mathematica.

We shall now describe how the different terms in the above series behave under the scaling argument described after eq. (3.19). As $A t_{K}$ is $t_{*} / r_{*}^{3}$ and since $t_{*} \sim t_{0} \Rightarrow A \sim t_{0} / r_{*}^{3}$. Using this scaling of $A$, we can see how different terms in the above equation scale.

For instance, the $A$ dependent term at $r^{8}$, after multiplying and dividing by $r_{*}^{2}$ and pluggin in the above scaling for $A$, is $\frac{A^{2}}{t_{0}^{3}} r^{8} \sim\left(r_{*}^{2} / t_{0}\right)\left(r / r_{*}\right)^{8}$. Similarly the $A^{4}$ term at $r^{14}$ is $\frac{A^{4}}{t_{0}^{5}} r^{14} \sim\left(r_{*}^{2} / t_{0}\right)\left(r / r_{*}\right)^{14}$. Whereas the $A^{2}$ dependent terms at $r^{10}, r^{12}$ and $r^{14}$ go as $\frac{A^{2}}{t_{0}^{5}} r^{10} \sim\left(r_{*}^{4} / t_{0}^{3}\right)\left(r / r_{*}\right)^{10}, \frac{A^{2}}{t_{0}^{7}} r^{12} \sim\left(r_{*}^{6} / t_{0}^{5}\right)\left(r / r_{*}\right)^{12}$ and $\frac{A^{2}}{t_{0}^{9}} r^{14} \sim\left(r_{*}^{8} / t_{0}^{7}\right)\left(r / r_{*}\right)^{14}$, respectively.

There are a few points which are noteworthy about these $A$ dependent terms which we describe below.

First and most importantly, this scaling argument shows that all the $A$ dependent terms are also suppressed as compared to the leading term in the series which is the first term.

The second point is that the terms at a particular order of $r$ can be compared with each other and the $A$ dependent terms are the ones which dominate within these terms, e.g, there are two terms at $r^{8}$ which are $\frac{r^{8}}{t_{0}^{7}} \sim\left(r_{*}^{8} / t_{0}^{7}\right)\left(r / r_{*}\right)^{8}$ and $\frac{A^{2}}{t_{0}^{3}} r^{8} \sim\left(r_{*}^{2} / t_{0}\right)\left(r / r_{*}\right)^{8}$. Since the former term has a higher power of $t_{0}$ in the denominator and since, $t_{0} \gg 1$, the latter term is dominant.

The final point is that at each order of $2 d_{i}$, a $A^{2 k}$ term appears where $k=1,2 \ldots$. This can be seen in the above case of $A d S_{5}$ where $A^{2}, A^{4}, A^{6}$ etc. appear at $r^{8}, r^{14}, r^{20}$, respectively. Keeping the dominant terms amongst each power of $r$ gives

$$
\begin{aligned}
t(r)= & t_{0}+\frac{1}{12 t_{0}} r^{2}-\frac{1}{432 t_{0}^{3}} r^{4}+\frac{1}{7776 t_{0}^{5}} r^{6}+\frac{A^{2}}{160 t_{0}^{3}} r^{8}-\frac{103 A^{2}}{160 \cdot 540 t_{0}^{5}} r^{10}+\frac{3943 A^{2}}{160 \cdot 540 \cdot 252 t_{0}^{7}} r^{12} \\
& +\frac{A^{4}}{160 \cdot 4 t_{0}^{5}} r^{14}-\frac{7 A^{4}}{160 \cdot 72 t_{0}^{7}} r^{16}+\frac{15011 A^{4}}{160 \cdot 540 \cdot 1120 t_{0}^{9}} r^{18}+\frac{91 A^{6}}{160 \cdot 880 t_{0}^{7}} r^{20} \\
& +\frac{8453 A^{6}}{22302720 t_{0}^{9}} r^{22}+\frac{493338049 A^{6}}{3653185536000 t_{0}^{11}} r^{24}+\frac{19 A^{8}}{56320 t_{0}^{9}} r^{26}+\ldots
\end{aligned}
$$

However at $r_{*}$ various terms become subleading using $A=\frac{t_{*}}{r_{*}^{3}}$ : e.g. we see that the $r_{*}^{10}$ and $r_{*}^{12}$ terms are suppressed by powers of $t_{0}$ and thus subleading compared with the $r_{*}^{8}$ term, all scaling as $A^{2}$. Now the $r_{*}^{14}$ term has scaling $\frac{t_{*}^{4}}{r_{*}^{12}} \frac{r_{*}^{14}}{t_{0}^{5}} \sim \frac{r_{*}^{2}}{t_{0}}$ which is the same as the $r^{8}$ term: the higher terms e.g. $r_{*}^{20}$ also has the same scaling, and so on. Thus the above series is further approximated as

$$
t(r)=t_{0}+\frac{r_{*}^{2}}{t_{0}}\left(\frac{1}{12}+\frac{1}{160}+\frac{1}{160 \cdot 4}+\frac{91}{160 \cdot 880}+\frac{19}{160 \cdot 352}+\ldots\right)
$$

Thus we recover (3.20), i.e. $t_{*}>t_{0}$ when $r_{*} \lesssim t_{0}$. 
All the arguments above hold for $A d S_{4}$ and $A d S_{7}$ Kasner as well. For $A d S_{4}$, we obtain

$$
\begin{aligned}
t(r)= & t_{0}+\frac{1}{12 t_{0}} r^{2}-\frac{1}{480 t_{0}^{3}} r^{4}+\frac{\left(13+1920 A^{2} t_{0}^{2}\right)}{120960 t_{0}^{5}} r^{6} \\
& -\frac{\left(125+44928 A^{2} t_{0}^{2}\right)}{17418240 t_{0}^{7}} r^{8}+\frac{\left(10543+6641280 A^{2} t_{0}^{2}+82944000 A^{4} t_{0}^{4}\right)}{19160064000 t_{0}^{9}} r^{10}+\ldots
\end{aligned}
$$

and for $A d S_{7}$

$$
\begin{aligned}
t(r)= & t_{0}+\frac{1}{15 t_{0}} r^{2}+\frac{1}{600 t_{0}^{3}} r^{4}+\frac{11}{135000 t_{0}^{3}} r^{6}-\frac{1}{200000 t_{0}^{7}} r^{8}+\frac{491}{1417500000 t_{0}^{9}} r^{10} \\
& +\frac{\left(-52891+3543750000 A^{2} t_{0}^{8}\right)}{128595600000000 t_{0}^{13}} r^{14}+\ldots
\end{aligned}
$$

\section{B Some details on calculating $S_{\text {bulk }}$}

Since any 2-dim metric is conformally flat, we have $d s^{2}=e^{f}\left(-d t^{2}+d r^{2}\right)$. If we now assume that the bulk matter can be modelled by a 2-dim CFT, we can obtain its entropy as in [2] by modifying the Calabrese-Cardy result [39, 40], in particular taking the ground state entanglement in flat space and incorporating the effects of the Weyl transformation $e^{f}$. The twist operator 2-point function scales under a conformal transformation as

$$
\left\langle\sigma\left(x_{1}\right) \sigma\left(x_{2}\right)\right\rangle_{e^{f} g}=\left.\left.e^{-\Delta_{n} f}\right|_{x_{1}} e^{-\Delta_{n} f}\right|_{x_{2}}\left\langle\sigma\left(x_{1}\right) \sigma\left(x_{2}\right)\right\rangle_{g}, \quad \Delta_{n}=\frac{c}{12} \frac{n^{2}-1}{n} .
$$

Since the partition function in the presence of twist operators scales as the twist operator 2-point function, the entanglement entropy becomes

$$
S_{e^{f} g}^{12}=-\lim _{n \rightarrow 1} \partial_{n}\left\langle\sigma\left(x_{1}\right) \sigma\left(x_{2}\right)\right\rangle_{e^{f} g}=S_{g}^{12}-\left.\frac{c}{6} \log e^{f}\right|_{x_{1}}-\left.\frac{c}{6} \log e^{f}\right|_{x_{2}}
$$

For the interval taken with one endpoint at the boundary, we essentially have a single twist operator and its 1-point function, using the boundary CFT prescription: this essentially restricts to just the single boundary in the bulk, say $x_{1}$, and effectively $c \rightarrow \frac{c}{2}$ above. We consider the interval $\left(r, r_{c}\right) \sim(r, 0)$ with endpoints being the extremal surface at $r$ and the boundary $r_{c} \sim 0$, the whole space being the half-line $(\infty, 0)$. We construct the replica space (with $w \equiv \tau+i x$ ) for this situation by gluing the $n$-sheets along the cut defined by the interval with twist operators at the single endpoint. To analyse the replica theory, we first map the half-line to a disc $|z| \leq 1$ via the uniformization map $z=\left(\frac{w-i l}{w+i l}\right)^{1 / n}$ which maps the boundary $w=0$ to $z=1$ and the endpoint at $x=l$ (so $w=i x=i l$ ) to $z=0$. There is a single twist operator at the endpoint $x=l$ (unlike two for a single interval in the full line). Taking the $z$-plane to be the $\operatorname{SL}(2)$ vacuum so $\langle T(z)\rangle=0$, we find the expectation value of the stress tensor $\langle T(w)\rangle_{R_{n}}$ via the Schwarzian for the $z(w)$ map above: this gives $\langle T(w)\rangle_{R_{n}}=\frac{c}{24}\left(1-\frac{1}{n^{2}}\right) \frac{(2 l)^{2}}{(w-i l)^{2}(w+i l)^{2}}$. Using BCFT Ward identities etc, this is equivalent to $\frac{\left\langle T(w) \sigma_{n}(i l)\right\rangle}{\left\langle\sigma_{n}(i l)\right\rangle}$ with $\left\langle\sigma_{n}(i l)\right\rangle=\frac{1}{(2 l)^{c(n-1 / n) / 12}}$. Then the replica partition function transforms as $\operatorname{Tr} \rho_{A}^{n} \sim\left\langle\sigma_{n}(i l)\right\rangle$ and $S_{A}=-\lim _{n \rightarrow 1} \partial_{n} \operatorname{Tr} \rho_{A}^{n}=\frac{c}{6} \log \frac{2 l}{\epsilon}$. Roughly this is like half the area (one endpoint rather than two for an interval in the full line space). So the expression in (4.1) is written for the 2-dim space with the boundary at $r=r_{c}$ and the QES at $(t, r)$. 
Since the conformal factor has nontrivial time-dependence, the conformal transformation above is nontrivial. In writing (4.1), we are making the nontrivial assumption that this formulation can be applied: this appears reasonable in the semiclassical region far from the singularity. However the presence of the Big-Crunch at $t=0$ as $e^{f} \rightarrow 0$ is expected to lead to a breakdown of this formulation, as we have stated in the text.

Open Access. This article is distributed under the terms of the Creative Commons Attribution License (CC-BY 4.0), which permits any use, distribution and reproduction in any medium, provided the original author(s) and source are credited.

\section{References}

[1] G. Penington, Entanglement wedge reconstruction and the information paradox, JHEP 09 (2020) 002 [arXiv: 1905.08255] [inSPIRE].

[2] A. Almheiri, N. Engelhardt, D. Marolf and H. Maxfield, The entropy of bulk quantum fields and the entanglement wedge of an evaporating black hole, JHEP 12 (2019) 063 [arXiv: 1905.08762] [INSPIRE].

[3] A. Almheiri, R. Mahajan, J. Maldacena and Y. Zhao, The Page curve of Hawking radiation from semiclassical geometry, JHEP 03 (2020) 149 [arXiv: 1908.10996] [INSPIRE].

[4] A. Almheiri, T. Hartman, J. Maldacena, E. Shaghoulian and A. Tajdini, The entropy of Hawking radiation, arXiv:2006.06872 [INSPIRE].

[5] T. Faulkner, A. Lewkowycz and J. Maldacena, Quantum corrections to holographic entanglement entropy, JHEP 11 (2013) 074 [arXiv: 1307.2892] [INSPIRE].

[6] N. Engelhardt and A.C. Wall, Quantum extremal surfaces: holographic entanglement entropy beyond the classical regime, JHEP 01 (2015) 073 [arXiv:1408.3203] [INSPIRE].

[7] S. Ryu and T. Takayanagi, Holographic derivation of entanglement entropy from AdS/CFT, Phys. Rev. Lett. 96 (2006) 181602 [hep-th/0603001] [INSPIRE].

[8] S. Ryu and T. Takayanagi, Aspects of holographic entanglement entropy, JHEP 08 (2006) 045 [hep-th/0605073] [INSPIRE].

[9] V.E. Hubeny, M. Rangamani and T. Takayanagi, A covariant holographic entanglement entropy proposal, JHEP 07 (2007) 062 [arXiv:0705.0016] [INSPIRE].

[10] M. Rangamani and T. Takayanagi, Holographic entanglement entropy, Lect. Notes Phys. 931 (2017) 1 [arXiv: 1609.01287] [INSPIRE].

[11] T.M. Fiola, J. Preskill, A. Strominger and S.P. Trivedi, Black hole thermodynamics and information loss in two-dimensions, Phys. Rev. D 50 (1994) 3987 [hep-th/9403137] [INSPIRE].

[12] Y. Chen, V. Gorbenko and J. Maldacena, Bra-ket wormholes in gravitationally prepared states, JHEP 02 (2021) 009 [arXiv:2007.16091] [INSPIRE].

[13] T. Hartman, Y. Jiang and E. Shaghoulian, Islands in cosmology, JHEP 11 (2020) 111 [arXiv: 2008.01022] [INSPIRE].

[14] C. Krishnan, Critical islands, JHEP 01 (2021) 179 [arXiv:2007.06551] [INSPIRE].

[15] M. Van Raamsdonk, Comments on wormholes, ensembles, and cosmology, arXiv: 2008.02259 [INSPIRE]. 
[16] V. Balasubramanian, A. Kar and T. Ugajin, Islands in de Sitter space, JHEP 02 (2021) 072 [arXiv:2008.05275] [INSPIRE].

[17] W. Sybesma, Pure de Sitter space and the island moving back in time, arXiv:2008.07994 [INSPIRE].

[18] S.R. Das, J. Michelson, K. Narayan and S.P. Trivedi, Time dependent cosmologies and their duals, Phys. Rev. D 74 (2006) 026002 [hep-th/0602107] [INSPIRE].

[19] A. Awad, S.R. Das, K. Narayan and S.P. Trivedi, Gauge theory duals of cosmological backgrounds and their energy momentum tensors, Phys. Rev. D 77 (2008) 046008 [arXiv:0711.2994] [INSPIRE].

[20] A. Awad, S.R. Das, S. Nampuri, K. Narayan and S.P. Trivedi, Gauge theories with time dependent couplings and their cosmological duals, Phys. Rev. D 79 (2009) 046004 [arXiv: 0807.1517] [INSPIRE].

[21] J.M. Maldacena, The large $N$ limit of superconformal field theories and supergravity, Adv. Theor. Math. Phys. 2 (1998) 231 [hep-th/9711200] [INSPIRE].

[22] S.S. Gubser, I.R. Klebanov and A.M. Polyakov, Gauge theory correlators from noncritical string theory, Phys. Lett. B 428 (1998) 105 [hep-th/9802109] [INSPIRE].

[23] E. Witten, Anti-de Sitter space and holography, Adv. Theor. Math. Phys. 2 (1998) 253 [hep-th/9802150] [INSPIRE].

[24] O. Aharony, S.S. Gubser, J.M. Maldacena, H. Ooguri and Y. Oz, Large N field theories, string theory and gravity, Phys. Rept. 323 (2000) 183 [hep-th/9905111] [INSPIRE].

[25] R. Bhattacharya, K. Narayan and P. Paul, Cosmological singularities and 2-dimensional dilaton gravity, JHEP 08 (2020) 062 [arXiv: 2006.09470] [INSPIRE].

[26] N. Engelhardt, T. Hertog and G.T. Horowitz, Holographic signatures of cosmological singularities, Phys. Rev. Lett. 113 (2014) 121602 [arXiv:1404.2309] [INSPIRE].

[27] N. Engelhardt, T. Hertog and G.T. Horowitz, Further holographic investigations of big bang singularities, JHEP 07 (2015) 044 [arXiv: 1503.08838] [INSPIRE].

[28] N. Engelhardt and G.T. Horowitz, Holographic consequences of a no transmission principle, Phys. Rev. D 93 (2016) 026005 [arXiv:1509.07509] [INSPIRE].

[29] N. Engelhardt and G.T. Horowitz, New insights into quantum gravity from gauge/gravity duality, Int. J. Mod. Phys. D 25 (2016) 1643002 [arXiv:1605.04335] [InSPIRE].

[30] B. Craps, Big bang models in string theory, Class. Quant. Grav. 23 (2006) S849 [hep-th/0605199] [INSPIRE].

[31] C.P. Burgess and L. McAllister, Challenges for string cosmology, Class. Quant. Grav. 28 (2011) 204002 [arXiv:1108.2660] [INSPIRE].

[32] K. Narayan, On aspects of 2-dim dilaton gravity, dimensional reduction and holography, arXiv:2010.12955 [INSPIRE].

[33] A. Strominger, Les Houches lectures on black holes, in NATO advanced study institute: Les Houches summer school, session 62: fluctuating geometries in statistical mechanics and field theory, (1994) [hep-th/9501071] [INSPIRE].

[34] A. Almheiri and J. Polchinski, Models of AdS $S_{2}$ backreaction and holography, JHEP 11 (2015) 014 [arXiv: 1402.6334] [inSPIRE]. 
[35] K. Narayan, T. Takayanagi and S.P. Trivedi, AdS plane waves and entanglement entropy, JHEP 04 (2013) 051 [arXiv: 1212.4328] [inSPIRE].

[36] N. Engelhardt and G.T. Horowitz, Entanglement entropy near cosmological singularities, JHEP 06 (2013) 041 [arXiv: 1303.4442] [INSPIRE].

[37] K.S. Kolekar and K. Narayan, On AdS 2 holography from redux, renormalization group flows and c-functions, JHEP 02 (2019) 039 [arXiv:1810.12528] [INSPIRE].

[38] R. Mahajan, A tutorial on entanglement island computations, in ICTS Bangalore string seminars, 26 August 2020.

[39] P. Calabrese and J.L. Cardy, Entanglement entropy and quantum field theory, J. Stat. Mech. 0406 (2004) P06002 [hep-th/0405152] [InSPIRE].

[40] P. Calabrese and J. Cardy, Entanglement entropy and conformal field theory, J. Phys. A 42 (2009) 504005 [arXiv:0905.4013] [INSPIRE].

[41] B. Czech, J.L. Karczmarek, F. Nogueira and M. Van Raamsdonk, The gravity dual of a density matrix, Class. Quant. Grav. 29 (2012) 155009 [arXiv:1204.1330] [INSPIRE].

[42] A.C. Wall, Maximin surfaces, and the strong subadditivity of the covariant holographic entanglement entropy, Class. Quant. Grav. 31 (2014) 225007 [arXiv:1211.3494] [INSPIRE].

[43] M. Headrick, V.E. Hubeny, A. Lawrence and M. Rangamani, Causality \& holographic entanglement entropy, JHEP 12 (2014) 162 [arXiv:1408.6300] [INSPIRE].

[44] A. Almheiri, R. Mahajan and J. Maldacena, Islands outside the horizon, arXiv:1910.11077 [INSPIRE].

[45] T. Hartman and J. Maldacena, Time evolution of entanglement entropy from black hole interiors, JHEP 05 (2013) 014 [arXiv: 1303.1080] [INSPIRE].

[46] K. Narayan, De Sitter future-past extremal surfaces and the entanglement wedge, Phys. Rev. D 101 (2020) 086014 [arXiv:2002.11950] [INSPIRE].

[47] S. Cooper, M. Rozali, B. Swingle, M. Van Raamsdonk, C. Waddell and D. Wakeham, Black hole microstate cosmology, JHEP 07 (2019) 065 [arXiv:1810.10601] [INSPIRE]. 